\title{
Recherches sur les suites régulières et les nombres de Bernoulli et d'Euler.
}

\author{
(Par Niels Nrelsen, à Copenhague.)
}

Dans un Mémoire récent intitulé : Sur les transcendantes élémentaires et les nombres de Bernoulli et d'Euler (*) j'ai étudié plusieurs polynomes entiers, dont les valeurs, pour des arguments particuliers, deviennent les nombres $B_{n}$ de Bernoulli, les coefficients des tangentes $T_{n}$ et les nombres $E_{n}$ d'Euler.

Par ce procédé j'ai donné de nombreuses expressions indépendantes d'un caractère très général des nombres susdits.

Or, parmi les polynomes qui possèdent la propriété susdite, la suite des fonctions de Bernoulur

$$
B_{0}(x), B_{1}(x), B_{2}(x), \ldots, B_{n}(x), \ldots
$$

et la suite des fonctions d'EuLek

$$
E_{0}(x), E_{1}(x), E_{2}(x), \ldots, E_{n}(x), \ldots
$$

semblent être les plus importantes.

En effet, prenons pour point de départ ces deux suites de polynomes entiers, il est possible de développer une théorie arithmétique et parfaitement élémentaire des nombres célèbres susdits; car une telle théorie n'exige que la connaissance de la formule binomiale qui correspond à un exposant positif entier.

Il est bien connu que la méthode classique, appliquée dans les recherches sur les nombres $B, T_{n}$ et $E_{n}$, savoir l'application de la fonction exponentielle et des fonctions trigonométriques est beaucoup simplifiée par la méthode symbolique découverte par A. Buissand $\left(^{* *}\right)$ et retrouvée par E. Lucas $\left.{ }^{* * *}\right)$.

(*) Annali di Matematica (3), t. 19, pp. 179-204; 1912.

(**) Quarterly Journal of Mathematics, t. 8, pp. 85-110, 1867; t.9, pp. 82-94, 154-177, 1868.

$\left.{ }^{* *}\right)$ Voir par exemple: Annali di Matematica (2), t. 8, pp. 56-79; 1877 et A. RADlcke: Die Recursionsformeln für die Berechnung der Bernoullischen und Eulerschen Zahlen. 1880. 
La méthode symbolique simplifie beaucoup les calculs, il est vrai, mais elle exige des calculs séparés pour chacune des trois classes des nombres $R_{n}, T_{n}$ et $E_{n}$.

Le point essentiel de ma méthode élémentaire est de remplacer les formules numériques susdites par des identités algébriques qui contiennent une variable complexe, ce qui nous donnera, en attribuant à la variable des valeurs spéciales convenables, d'un coup de main, toutes les formules connues de ce genre et un grand nombre d'autres.

Dans deux Mémoires intitulés: Recherches sur les nombres de Bernoulli (*) et Recherches sur les fonctions et les nombres de Bernoulli et d'Euler (**) dont le dernier n'est publié pas encore j'ai donné les fondements de la théorie élémentaire susdite.

Le but du Mémoire présent est l'étude d'un seul point de ma théorie générale, savoir le développement des classes des formules récursives pour les $B_{n}, T_{n}$ et $E_{n}^{\prime}$, formules 'très remarquables, d'un caractère presque arbitraire.

A cet effet, il est nécessaire de considérer quelques propriétés fondamentales des fonctions de Bernoulut et d'Euner.

\section{PREMIÈRE PARTIE.}

\section{Les polynomes réguliers.}

\section{Déhinitions et proprif́tés fondamentales.}

Je définis les fonctions de Bennoular à l'aide des deux équations fonctionnelles $\left(^{* * *}\right)$

$$
B_{n}^{\prime}(x)=B_{n-1}(x), \quad B_{n}(x)-B_{n}(x-1)=\frac{x^{n-1}}{(n-1) !},
$$

(*) Mémoires de l'Académie Royale des Sciences et des Lettres de Danemark, 1913.

(**) Annales de l'École Normale; Paris.

$\left({ }^{* * * *}\right)$ Comparer par exemple la définition beaucoup plus compliquée donnée par A. Bencen dans les Acta mathematica, t. 14, p. 251; 1890-91. 
où il faut admettre $n \geqq 1$; en étudiant l'équation aux différences finies (1) je trouve pour les $B_{n}(x)$ les expressions suivantes

$$
\left.\begin{array}{l}
B_{0}(x)=1, \quad B_{1}(x)=x+\frac{1}{2} \\
B_{n}(x)=\frac{x^{n}}{n !}+\frac{1}{2} \cdot \frac{x^{n-1}}{(n-1) !}+\sum_{s=1}^{\sum_{2}} \frac{(-1)^{s-1} B_{s} x^{n-2 s}}{(2 s) !(n-2 s) !}
\end{array}\right\}
$$

et je démontre que les nombres rationnelles $B_{n}$ sont tous positifs. Cette introduction des nombres de Bernoulur coïncide avec celle d'Eulen $\left(^{*}\right)$.

De même je définis les fonctions d'Eucer à l'aide des équations aux différences finies

$$
E_{n}(x)+E_{n}(x-1)=\frac{x^{n}}{n !}, \quad n \geqq 0
$$

ce qui donnera les expressions suivantes

$$
\left.\begin{array}{l}
E_{0}(x)=\frac{1}{2} \\
E_{n}(x)=\frac{1}{2} \cdot \frac{x^{n}}{n !}+\sum_{s=1}^{\leqq \frac{n+1}{2}} \frac{(-1)^{s-1} T_{s} x^{n-2 s+1}}{(2 s-1) !(n-2 s+1) ! 2^{2 s}},
\end{array}\right\}
$$

et je démontre de cette manière que les nombres $T_{n}$ sont des positifs entiers, pairs à l'exception de $T_{1}=1$.

Il est évident que les fonctions $E_{n}(x)$ satisfont, pour $n \geqq 1$, à l'équation fonctionnelle

$$
E_{n}^{\prime}(x)=E_{n-1}(x)
$$

Combinons maintenant les expressions (2) et (4) et les équations aux différences finies (1) et (3), il est évident que les fonctions $B_{n}(x)$ et $E_{n}(x)$ satisfont aux équations fonctionnelles

$$
(-1)^{n} B_{n}(-x-1)=B_{n}(x), \quad(-1)^{n} E_{n}(-x-1)=E_{n}(x) .
$$

De plus, il saute aux yeux que les deux formules (2) et (4) nous permettent de déterminer, pour tous les $n$, les valeurs numériques $B_{n}(0)$ et

(*) Institutiones calculi differentialis, pp. 409-410, 421-422. Saint-Pétersbourg, 1755. 
$E_{n}(0)$; nous trouvons pour les fonctions de Bernoultr

$$
\left.\begin{array}{l}
B_{0}(0)=1, \quad B_{1}(0)=\frac{1}{2} \\
B_{2 n+1}(0)=0, \quad B_{2 n}(0)=\frac{(-1)^{n-1} B_{n}}{(2 n) !}, n \geqq 1
\end{array}\right\}
$$

et pour les fonctions d'Eucer

$$
\left.\begin{array}{l}
E_{0}(0)=\frac{1}{2}, \quad E_{2 n}(0)=0, \quad n \geqq 1 \\
E_{n_{n+1}}(0)=\frac{(-1)^{n} T_{n+1}}{(2 n+1) ! 2^{2 n+2}}, \quad n \geqq 0 .
\end{array}\right\}
$$

Quant aux valeurs, numériques qui correspondent à $x=-\frac{1}{2}$, nous considérons les deux identités évidentes

$$
\begin{aligned}
& B_{n}(x)=\mathscr{Q}^{n-1}\left(B_{n}\left(\frac{x}{2}\right)+B_{n}\left(\frac{x-1}{2}\right)\right) \\
& E_{n}(x)=2^{n}\left(B_{n+1}\left(\frac{x}{2}\right)-B_{n+1}\left(\frac{x-1}{2}\right)\right),
\end{aligned}
$$

cas particuliers des formules de RaABe $\left(^{*}\right)$.

Cela posé, nous aurons pour les fonctions de BerNouldi

$$
\left.\begin{array}{c}
B_{0}\left(\begin{array}{c}
1 \\
2
\end{array}\right)=1, \quad B_{3 n}\left(-\frac{1}{2}\right)=\frac{(-1)^{n}\left(2^{2 n}-2\right) B_{n}}{(2 n) ! 2^{2 n}}, n \geqq 1 \\
B_{2 n+1}\left(-\frac{1}{2}\right)=0, \quad n \geqq 0
\end{array}\right\}
$$

et pour les fonctions d'EuLER

$$
\begin{gathered}
E_{0}\left(-\frac{1}{2}\right)=\frac{1}{2}, \quad E_{2_{n}}\left(-\frac{1}{2}\right)=\frac{(-1)^{n} E_{n}}{(2 n) ! 2^{2 n+1}}, n \geqq 1 \\
E_{2 n+1}\left(-\frac{1}{2}\right)=0 .
\end{gathered}
$$

(*) Jownal de Crelle, t. 42, pp. 356-357; 1851. 
Je définis précisément de cette manière les nombres $E_{n}$ d'EuLER, et on voit que cette introduction des $E_{n}$ coïncide avec celle d'Euler $\left(^{*}\right)$.

Remarquons en passant que la formule (10) donnera immédiatement la formule d'EuLER $(* *)$

$$
T_{n}=\frac{2^{2 n}\left(2^{2 n}-2\right) B_{n}}{2 n}
$$

Généralisons maintenant les équations fonctionnelles (6) en étudiant le polynome entier du degré $n$ par rapport à $x$

$$
f_{n}(x)=\sum_{s=0}^{s=n} a_{n, s} x^{n-s}
$$

qui est assujetti à satisfaire à l'équation fonctionnelle

$$
(-1)^{n} f_{n}(-x-1)=f_{n}(x) \text {. }
$$

Nous désignons dans ce qui suit comme réguliers tous les polynomes de ce genre.

Remarquons que l'équation (15) donnera immédiatement

$$
f_{n}(x-1)=(-1)^{n} f_{n}(-x),
$$

nous aurons, en vertu de (14), ces deux équations aux différences finies

$$
\begin{aligned}
& f_{n}(x)-f_{n}(x-1)=2 . \sum_{s=0} a_{n, 2 s+1} x^{n-2 s-1} \\
& f_{n}(x)+f_{n}(x-1)=2 . \sum_{s=0} a_{n, 2 s} x^{n-2 s}
\end{aligned}
$$

ce qui donnera, en vertu de (1) et (3), ces deux développements de $f_{n}(x)$

$$
\begin{aligned}
& \frac{1}{2} f_{n}(x)=K_{n}+\sum_{s=1}^{\leqq \frac{n-1}{n}}(n-2 s-1) ! a_{n, 2 s+1} B_{n-2 s}(x), \\
& \frac{1}{2} f_{n}(x)=\sum_{s=0}(n-2 s) ! a_{n, 2 s} E_{n-2 s}(x),
\end{aligned}
$$

(*) Opuscula Analytica, t. II, pp. 269-270. Saint-Pétersbourg, 1785.

(**) Loc. cit., p. 273. 
où, dans (16), $K_{2, n}$ est une constante, tandis que nous aurons, en vertu de (15), $K_{2 n+1}=0$.

Les deux développements (14) et (17) montrent clairement que le polynome $f_{n}(x)$ qui satisfait à l'équation fonctionnelle (15) est parfaitement déterminé si nous connaissons ou tous les coefficients $a_{n, 2 s}$ ou tous les $a_{n, 2, s+1}$. Soit $n$ un nombre pair, le coefficient $a_{n, n}$ peut toujours être choisi arbitrairement.

Nous nous bornerons ici à ces remarques sur l'équation fonctionnelle (15) que j’ai étudiée assez amplement dans mes deux Mémoires susdits.

Cependant, il nous reste encore de modifier les développements (16) et (17).

A cet effet, nous désignons par

$$
a_{0} \alpha_{1} \alpha_{2} \ldots \alpha_{n} \ldots
$$

une suite infinie qui satisfait à la seule condition $\left|a_{0}\right|>0$, mais qui est du reste parfaitement arbitraire, puis nous posons pour tous les $n$

$$
f_{n}(x)=\sum_{s=0}^{s=n} \frac{a_{s} x^{n-s}}{(n-s) !} .
$$

Cela posé, il est évident que $f_{n}(x)$ est, pour tous les $n$, du degré $n$ par rapport à $x$ et que les éléments de la suite infinie

$$
f_{0}(x), f_{:}(x), f_{2}(x), \ldots, f_{n}(x), \ldots
$$

satisfont, pour $n \geqq 1$, à l'équation ronctionnelle

$$
f_{n}^{\prime}(x)=f_{n-1}(x)
$$

Supposons maintenant que tous les éléments de la suite infinie (20) soient des polynomes réguliers, saroir qu'ils satisfassent pour tous les $n$ à l'équation fonctionnelle (15), nous désignons comme régulière la suite (20), et nous indiquons par le symbole $\left[f_{n}(x), a_{n}\right]$ que les polynomes $f_{n}(x)$ sont, pour tous les $n$, à déterminer par la formule (19). Nous désignons la suite (18) comme la base de la suite régulière (20).

Soit $\left[f_{n}(x), a_{n}\right]$ une suite régulière quelconque, nous aurons, en vertu de (16) et (17), pour tous les $n$, les développements suivants

$$
\begin{aligned}
& \frac{1}{2} f_{n}(x)=\sum_{s=0}^{\leq \frac{n}{2}} a_{2 s+1} B_{n-2 s}(x) \\
& \frac{1}{2} f_{n}(x)=\sum_{s=0} a_{n s} E_{n-2 s}(x) ;
\end{aligned}
$$


c'est-à-dire que la suite régulière $\left[f_{n}(x), a_{n}\right]$ est parfaitement déterminée, pourru que tous les éléments $a_{2 s}$ ou tous les éléments $a_{2 s+1}$ de sa base soient connus.

On détermine immédiatement les bases qui correspondent aux suites harmoniques formées des $B_{n}(x)$ ou des $E_{n}(x)$.

II. Le porynome RÉGULter le PLUS gF́nélial.

Pour étudier, au point de vue des zéros aussi, un polynome, régulier nous choisissons $n$ nombres complexes

$$
\alpha_{1} \alpha_{2} \alpha_{3} \ldots \alpha_{n}
$$

qui satisfont, pour $1 \leqq s \leqq n$, aux conditions

$$
\alpha_{s}+\alpha_{n-s+1}=m
$$

où $m$ désigne un nombre complexe différent de zéro mais quelconque du reste. De plus, nous supposons les nombres (1) aussi arbitraires que les conditions (2) le permettent.

Soit $n=2 q$ ou $n=2 q+1$, il est par conséquent possible de choisir précisément $q$ des nombres $\alpha_{s}$ parfaitement arbitraires; dans le demier cas, où $n$ est supposé impair, l'ensemble (1) contient l'élément $\frac{1}{2} m$.

Cela posé, il est évident que le polynome du degré $n$ par rapport à $x$

$$
F_{n}(x)=\left(x+\frac{\alpha_{1}}{m}\right)\left(x+\frac{\alpha_{2}}{m}\right) \cdots\left(x+\frac{\alpha_{n}}{m}\right)
$$

est régulier; car nous aurons, en vertu de (1), pour $1 \leqq s \leqq n$

$$
-x-1+\frac{\alpha_{s}}{m}=-\left(x+\frac{\alpha_{n-s+1}}{m}\right) .
$$

Posons ensuite pour abréger

$$
\left(x+\alpha_{1}\right)\left(x+\alpha_{2}\right) \ldots\left(x+\alpha_{n}\right)=\sum_{s=0}^{s=n} A_{n, s} x^{n-s},
$$


nous aurons évidemment

$$
F_{n}(x)=\sum_{s=0}^{s=n} \frac{A_{n, s}}{m^{s}} \cdot x^{n-s}
$$

ce qui donnera, en vertu des formules (16) et (17) du paragraphe I, ces deux développements

$$
\begin{aligned}
& \frac{1}{2} F_{n}(x)=K+\sum_{s=0}^{\sum^{n}} \frac{(n-2 s-1) ! A_{m, 2,+1}}{m^{2 s+1}} B_{n-2 s}(x) \\
& \frac{1}{2} F_{n}(x)=\sum_{s=0} \frac{(n-2 s) ! A_{n, s s}}{m^{2 s}} E_{n-2 s}(x) .
\end{aligned}
$$

Remarquons encore que l'équation fonctionnelle (15) du paragraphe I donnera pour $0 \leqq p \leqq m$

$$
(\stackrel{1}{\prime})^{p} F_{n}^{(n-p)}(-x-1)=F_{n}^{(n-p)}(x),
$$

il est très facile de déduire plusieurs groupes de relations entre les coefficients $A_{i, p}$.

Formules de première espèce. Posons dans (8) $x=0$, nous aurons en vertu de $(\dot{5})$, et pourvu que $1 \leqq p \leqq n$

$$
\left(1-(-1)^{p}\right) A_{n, p}=\sum_{s=0}^{s=p-1}(-1)^{s}\left(\begin{array}{l}
n-s \\
p-s
\end{array}\right) m^{p-s} A_{n, *},
$$

d'où, en remplaçant $p$ par $2 p+1$ respectivement par $2 p+2$, ces deux autres formules

$$
\begin{gathered}
2 A_{n, 2, p+1}=\sum_{s=0}^{s=2 p}(-1)^{s}\left(\begin{array}{c}
n-s \\
2 p-s+1
\end{array}\right) m^{2 p-2 s+1} A_{n, s} \\
(n-2 p-1) A_{n, 2, p+1}=\sum_{s=0}^{s=2 p}(-1)^{s}\left(\begin{array}{c}
n-s \\
2 p-s+2
\end{array}\right) m^{2 p-2 s+1} A_{n, s} .
\end{gathered}
$$

Posons ensuite dans (8) $2 p+1$ au lieu de $p$ et $x=-\frac{1}{2}$, nous aurons

$$
\sum_{s=0}^{s=\sum_{p+1}}(-1)^{s}\left(\begin{array}{c}
n-s \\
2 p-s+1
\end{array}\right) 2^{s} m^{2 p-s+1} A_{n, s}=0 .
$$

Il est digne de remarque que les trois formules (10), (11) et (12) sont équivalentes. 
Formules de deuxième espèce. Introduisons aux seconds membres de (6) et (7) les expressions obtenues pour les fonctions de Bernoulur ou d'Euler, puis ordonnons suivant des puissances descendantes de $x$, nous aurons respectivement

$(-1)^{p}\left(\frac{A_{n, 2 p+1}}{n-2 p}-\frac{m}{2} A_{n, 2 p}\right)=\sum_{s=0}^{s=p-1}(-1)^{s}\left(\begin{array}{c}n-2 s-1 \\ 2 p-2 s\end{array}\right) \frac{m^{2 p-2 s} A_{m, 2 s+1} B_{p-s}}{n-2 p}$,

où il faut admettre $1 \leqq p \leqq \frac{n-1}{2}$, et

$$
(-1)^{y} A_{w, 2 p+1}=\sum_{s=0}^{s=p}(-1)^{*}\left(\begin{array}{c}
n-2 s \\
2 p-2 s+1
\end{array}\right)\left(\frac{m}{2}\right)^{2 p-2 s+1} A_{n, 2 s} T_{p-s+1}
$$

formule qui est valable pour $0 \leqq p \leqq \frac{n-1}{2}$.

Il saute aux yeux que les denx systèmes des formules (13) et (14) sont équivalents entre eux et avec les trois précédents. Les réductions des seconds membres des formules de première espèce obtenues en introduisant les $B_{n}$ ou les $T_{n}$ sont très remarquables.

Posons, dans (14) $p=0$, nous aurons le résultat évident

$$
A_{i, 1}=\alpha_{1}+\alpha_{2}+\alpha_{3}+\cdots+\alpha_{32}=\frac{m \cdot n}{2} .
$$

Formule de troisième espèce. Supposons $n$ pair, remplaçons $n$ par $2 n$, puis posons pour abréger

$$
P_{2 n}=\left(2 \alpha_{1}-m\right)\left(2 \alpha_{2}-m\right) \ldots\left(2 \alpha_{2 n}-m\right),
$$

nous aurons évidemment

d'où, en vertu de (5),

$$
P_{2 n}=(2 m)^{2 n} F_{2 n}\left(-\frac{1}{2}\right)
$$

$$
P_{2 n}-2^{2 n} A_{2 n, 2 n}=\sum_{s=10}^{s=2 n-1}(-1)^{s} 2^{\kappa} m^{2 n-s} A_{2 n, s} .
$$

Posons ensuite, dans (7), $x=-\frac{1}{2}$, il en résulte, en vertu de la définition des nombres d'EuLer, savoir la formule (12) du paragraphe I,

$$
(-1)^{n}\left(P_{2 n}-2^{2 n} A_{2 n, 2 n}\right)=\sum_{s=0}^{s=n-1}(-1)^{s} 2^{2 s} m^{2 n-2 s} A_{2 n, 2 s} E_{n-s} .
$$


Posons de même, dans $(6), x=0, x=-\frac{1}{2}$, puis soustrayons les deux résultats ainsi obtenus, la formule eulérienne (13) du paragraphe I donnera

$$
(-1)^{n}\left(P_{2 n}-2^{2 n} A_{2 n, 2 n}\right)=\sum_{s=0}^{s=n-1}(-1)^{s}\left(\frac{m}{2}\right)^{2 n-2 s-1} 2^{2 s+1} A_{2 n, 2 s+1} T_{n-s},
$$

formule qui ne contient pas la constante $K_{2 n}$.

Les trois dernières formules sont équivalentes aussi; remarquons la simplification du second membre de (18) obtenue en introduisant les $E_{n}$ et les $T_{n}$.

Appliquons les formules (10) et (19), nous aurons immédiatement le théorème suivant:

1. Supposons que tous les nombres $x_{s}$ soient des entiers, la quantité $m$ et les coefficients $A_{n, s}$ ont la nême propriété, et nous aurons dans ce cas les congruences suivantes

$$
\begin{aligned}
& 2 A_{n, 2_{2}+1} \equiv 0(\bmod m), \quad 0 \leqq p \leqq \frac{n-1}{2} \\
& P_{2_{n}}-2^{2 n} A_{2 n, 2 n} \equiv 0\left(\bmod m^{2}\right), \quad n \geq 1
\end{aligned}
$$

\section{Cas particuliers essentiels.}

Il nous semble utile, à cause de nos recherches suivantes, de discuter ici plus amplement certains cas particuliers des formules générales que nous venons de développer.

Premier cas particulier. Soit l'ensemble des $\alpha_{s}$ les nombres naturels

$$
1,2,3, \ldots, n-1 \text {, }
$$

nous avons à poser $m=n$ et à remplacer $n$ par $n-1$. Posons ensuite comme ordinairement

$$
(x+1)(x+2) \ldots(x+n-1)=\sum_{s=0}^{s=n-1} C_{n}^{s} x^{n-s-1},
$$

où les nombres $C_{n}$ sont les coefficients de la factorielle du rang $n$, nous aurons par conséquent

$$
A_{n, s}=C_{n}^{s}
$$


Quant à $P_{2 n}$, nous aurons en remplaçant $n$ par $2 n+1$,

$$
P_{2 n}=(-1)^{n}[1.3 .5 \ldots(2 n-1)]^{2} \text {. }
$$

L'introduction de ces deux valeurs dans les formules générales du paragraphe II étant évidente, nous nous bornons à indiquer les deux congruences ainsi obtenues

$$
\begin{gathered}
2 C_{n}^{2 p+1} \equiv 0(\bmod n), \quad 0 \leqq p \leqq \frac{n-2}{2} \\
{[1.3 .5 \ldots(2 n-1)]^{2} \equiv(-1)^{n} 2^{2 n}(2 n) !\left(\bmod (2 n+1)^{2}\right), \quad n \geqq 1,}
\end{gathered}
$$

d'où, en supposant que $2 n+1$ soit un nombre premier $\left(^{*}\right)$,

$$
1.3 .5 \ldots(2 n-1) \equiv(-1)^{2 n} 2^{3 n} \cdot n !\left(\bmod (2 n+1)^{2}\right) \text {. }
$$

Dans ce qui suit nous avons à appliquer les deux sommes de puissances numériques

$$
s_{q}(n)=\sum_{r=1}^{r=n} r^{q}, \quad \sigma_{q}(n)=\sum_{r=0}^{r=n-1}(-1)^{r}(n-r)^{q} .
$$

Remarquons en passant que nous pouvons aussi, dans ce cas, choisir l'ensemble des $\alpha_{*}$ comme les nombres

$$
0,1,2,3, \ldots, n
$$

de sorte que nous avons à poser $m=n$ et à remplacer $n$ par $n+1$.

Deuxième cas particulier. Soit l'ensemble des $\alpha_{s}$ les $n$ nombres impairs

$$
1,3, \overline{5}, \ldots, 2 n-1 \text {, }
$$

nous avons à poser $m=2 n$; nous trouvons immédiatement

$$
P_{2 n}=(-1)^{n} 2^{2 n}[1.3 .5 \ldots(2 n-1)]^{2} .
$$

Posons ensuite pour abréger

$$
(x+1)(x+3) \ldots(x+2 n-1)=\sum_{s=0}^{s=n} D_{n}^{s} x^{n-s}
$$

nous aurons de même

$$
A_{n, s}=D_{n}^{s}
$$

(*) La congruence (6) est valable encore pour le module $(2 u+1)^{3}$. 
Cela posé, nous obtenons, daus ce cas, les deux congruences

$$
\begin{gathered}
2 D_{n}^{2 p+1} \equiv 0(\bmod 2 n), 0 \leqq p \leqq \frac{n-1}{2} \\
(-1)^{n} 2^{2 n}[1.3 .5 \ldots(2 n-1)]^{2} \equiv 2^{2 n} \cdot 1.3 .5 \ldots(4 n-1)\left(\bmod 16 n^{2}\right) .
\end{gathered}
$$

Dans ce qui suit, nous avons à appliquer les deux sommes de puissances numériques

$$
t_{u}(n)=\sum_{s=0}^{s=n-1}(2 n-2 s-1)^{x}, \quad \tau_{q}(n)=\sum_{s=0}^{s=n-1}(-1)^{n}(2 n-2 s-1)^{q},
$$

formées des nombres (9).

Troisième cas particulier. Soit $m>2$ un entier quelconque du reste, et soit $n=\varphi(m)$ la fonction introduite dans la théorie des nombres par EuLER, les $u$ nombres

$$
\alpha_{1} \alpha_{2} \alpha_{3} \ldots \alpha_{n}
$$

plus petits que $m$ et premiers avec $m$ satisfont encore à la condition (2) $d u$ paragraphe II; dans ce cas $n$ est toujours un nombre pair, savoir $n=2 \mu$.

Supposons $m$ impair, hypothèse qui est la plus intéressante, la moitié des nombres (15) sont pairs, les autres $\mu$ impairs. Désignons par

les nombres pairs, par

$$
\gamma_{1} \gamma_{2} \ldots \gamma_{*}
$$

$$
\delta_{1} \delta_{2} \ldots \delta_{\mu}
$$

les nombres impairs contenus dans l'ensemble (15), nous aurons

$$
P_{n}=(-1)^{\mu}\left(\delta_{1} \delta_{2} \ldots \delta_{\mu}\right)^{2}
$$

ce qui donnera la congruence

$$
(-1)^{\mu} \delta_{1} \delta_{2} \ldots \delta_{\mu} \equiv \mathscr{Q}^{n} \gamma_{1} \gamma_{2} \ldots \gamma_{\mu}\left(\bmod m^{2}\right)
$$

Dans le cas, où $m$ est supposé pair, tous les nombres $\alpha_{s}$ sont impairs; dans ce cas il n'est pas possible de donner généralement à la congruence (22) du paragraphe II une forme si simple que (19).

Dans ce qui suit nous avons à appliquer les sommes de puissances formées des nombres (15)

$$
s_{q}=\alpha_{1}^{q}+\alpha_{2}^{q}+\cdots+i_{n}^{q}
$$


sommes pour lesquelles A. Thacker (*) a indiqué des expressions remarquables.

Soit $m$ un nombre impair, nous posons encore

$$
\sigma_{q}=\sum_{s=1}^{s=n}(-1)^{\alpha_{s}} \alpha_{s}^{q}
$$

et en appliquant la définition (17)

$$
t_{q}=\sum_{s=1}^{s=\sum_{s}^{\mu}} \delta_{s}^{q}, \quad \tau_{q}=\sum_{s=1}^{s=\mu}(-1)^{\frac{\delta_{s}-1}{2}} \delta_{s}^{q} ;
$$

on voit que ces sommes de puissances coïncident avec les précedentes dans le cas où $m$ est un nombre premier impair.

Remarquons du reste qu'il est très facile de déduire pour les $\sigma_{q}$ des expressions analogues à celles données par Thacker pour les $s_{q}$ et que ces expressions sont des conséquences immédiates de réflexions suivantes:

Soit $m$ décomposé dans ses facteurs premiers comme suit:

$$
m=p_{1}^{\alpha_{1}} p_{\mathrm{Z}}^{\alpha_{2}} \cdots p_{r}^{\alpha_{r}},
$$

nous aurons

$$
\varphi(m)=m\left(1-\frac{1}{p_{1}}\right)\left(1-\frac{1}{p_{2}}\right) \cdots\left(1-\frac{1}{p_{r}}\right)
$$

Désignons ensuite par

$$
m_{1}^{(q)}, m_{\mathrm{q}}^{(q)}, \ldots, m_{t_{q}}^{(q)}, \quad t_{q}=\left(\begin{array}{l}
r \\
q
\end{array}\right)
$$

les positifs entiers obtenus en divisant $m$ par $q$ de ses facteurs premiers, par

$$
\pi_{1}^{(q)}, \pi_{q}^{(q)}, \ldots, \pi_{\tau_{q}}^{(q)}
$$

les produits correspondants des $q$ facteurs premiers de $m$, nous aurons toujours

$$
\pi_{s}^{(q)} m_{s}^{(q)}=m, \quad 1 \leqq s \leqq t_{\psi}
$$

Cela posé, il est évident que la formule (24) se présente sous cette autre

(*) Journal de Crelle, t. 40, pp. 89-90; 1850. Nowvelles Annales, t. 10, pp. 324-328; 1851 . BunET, duns les Comptes rendus, t. 32, pp. 918-921, 1851, a résoln le même problème; comparez E. Prounet, dans les Nouvelles Aunales, t. 10, pp. 398-330; 1851. 
forme aussi

$$
\varphi(m)=m+\sum_{s=1}^{s=r}(-1)^{s} \sum_{\nu=1}^{\nu=t_{v}} m m_{\nu}^{(s)}
$$

Dans ce qui suit, nous avons à donner des applications intéressantes de la méthode de Thacker.

\section{DEUXIÈME PARTIE.}

\section{Suites régulières très générales.}

\section{Formules fondamentiales.}

Prenons pour point de départ les nombres $\alpha_{s}$ appliqués dans le paragraphe II, il est très facile de construire des suites régulières d'une forme très générale.

A cet effet, nous avons à adjoindre aux nombres $\alpha_{s}$ un ensemble correspondant

$$
\beta_{1} \beta_{2} \beta_{3} \ldots \beta_{n}
$$

de sorte que $\beta_{s}$ correspond à $\alpha_{s}$ et que nous aurons pour $1 \leqq s \leqq n$

$$
\beta_{s}=(-1)^{\varepsilon} \beta_{n-s+1},
$$

où $\varepsilon$ est un nombre fixe. Du reste les nombres $\beta_{s}$ sont à choisir aussi arbitraires que ces conditions le permettent.

Cela posé, il est évident que les polynomes

$$
g_{p}(x)=\frac{1}{p !} \cdot \sum_{s=1}^{s=n} \beta_{s}\left(x+\frac{\alpha_{s}}{m}\right)^{p}
$$

forment une suite régulière; car nous aurons évidemment

$$
\beta_{s}\left(-x-1+\frac{\alpha_{s}}{m}\right)^{p}=(-1)^{p+\varepsilon} \beta_{n-s+1}\left(x+\frac{\alpha_{n-s+1}}{m}\right)^{p} .
$$


On voit que le degré du polynome $g_{p}(x)$ ne peut jamais dépasser $p$ et que la base de la suite régulière susdite est intimément liée avec les sommes

$$
\gamma_{q}=\sum_{s=1}^{s=n} \beta_{s} \alpha_{s}^{q}
$$

Supposons

$$
\gamma_{0}=\gamma_{1}=\gamma_{2}=\cdots=\gamma_{r-1}=0, \quad\left|\gamma_{r}\right|>0
$$

la fonction

$$
\Phi_{q}(x)=\frac{1}{(q+r) !} \cdot \sum_{s=1}^{s=n} \beta_{s}\left(x+\frac{\alpha_{s}}{m}\right)^{q+\cdots}
$$

est toujours, même pour $r=0$, un polynome régulier du degré $q$, ce qui donnera, en vertu de (3),

$$
(-1)^{q} \Phi_{q}(-x-1)=(-1)^{q+r+\varepsilon} \Phi_{q}(-x-1)=\Phi_{q}(x),
$$

d'où la proposition suivante.

I. Supposons remplies les conditions susdites, la somme

est toujours un nombre pair.

$$
r+\varepsilon
$$

Posons maintenant pour abréger

$$
a_{\rho}=\gamma_{i+\rho}
$$

nous aurons, en vertu de $(6)$,

$$
\Phi_{q}(x)=\sum_{s=0}^{s=q} \frac{\alpha_{s}}{(r+s) ! m^{r+s}} \cdot \frac{x^{q-s}}{(q-s) !},
$$

de sorte que les formules (22) et (23) du paragraphe I donnent ces deux développements :

$$
\begin{aligned}
& \frac{1}{2} \Phi_{q}(x)=\sum_{s=0} \frac{\frac{q}{q}}{(r+2 s+1) ! m^{r+2 s+1}} B_{q-2 s}(x) \\
& \frac{1}{2} \Phi_{q}(x)=\sum_{s=0} \frac{a_{2 s+1}}{(r+2 s) ! m^{r+2 s}} E_{q-2 s}(x) .
\end{aligned}
$$

Les dérivées du polynome $\Phi_{q}(x)$ étant des fonctions de même genre, nous trouvons évidemment les formules analogues à celles du paragraphe II en substituant simplement, dans les développements divers de $\Phi_{q}(x)$, les valeurs 
particulières de $x$ en question. De ce procédé nous trouvons les formules suivantes:

Formules de première espèce:

$$
\begin{gathered}
\left(1-(-1)^{q}\right) a_{q}=\sum_{s=0}^{s=q-1}(-1)^{s}\left(\begin{array}{c}
r+q \\
r+s
\end{array}\right) m^{q-s} a_{s} \\
2 a_{2 q+1}=\sum_{s=0}^{s=2 q}(-1)^{s}\left(\begin{array}{c}
r+2 q+1 \\
r+s
\end{array}\right) m^{2 q-s+1} a^{s} \\
(r+2 q+2) a_{2 q+1}=\sum_{s=0}^{s=2 q}(-1)^{s}\left(\begin{array}{c}
r+9 q+2 \\
r+s
\end{array}\right) m^{2 q-s+1} a_{s} \\
2^{2 q+1} a_{2 q+1}=\sum_{s=0}^{s=2 q}(-1)^{s} \cdot\left(\begin{array}{c}
r+2 q+1 \\
r+s
\end{array}\right) 2^{s} m^{2 q-s+1} a_{s}
\end{gathered}
$$

dans (11) il faut supposer $q \geqq 1$; posons $q=1$, nous aurons

$$
a_{1}=\frac{m(r+1)}{2} a_{0} \text {. }
$$

Formules de deuxième espèce:

$$
\left.\left.\begin{array}{l}
(-1)^{q}\left(a_{2 q+1}-\frac{m(r+2 q+1)}{2} a_{2 q}\right)= \\
\quad=\sum_{s=0}^{s=q-1}(-1)^{s}\left(\begin{array}{c}
r+2 q+1 \\
r+2 s+1
\end{array}\right) m^{2 q-2 s} a_{2 s+1} B_{r-s}
\end{array}\right\}\right)
$$

dans (16) il faut supposer également $q \geqq 1$.

Formules de troisième espèce. Posons pour abréger

$$
b_{2 q}=\frac{1}{2 r} \sum_{s=1}^{s=n z} \beta_{s}\left(2 \alpha_{s}-m\right)^{r+2 q}=(r+2 q) ! 2^{2 q} m n^{r+2 q} \Phi_{2 q}\left(-\frac{1}{2}\right),
$$

nous aurons les formules suivantes valables pourvu que $q \geqq 1$ :

$$
\begin{aligned}
& b_{2 q}-2^{2 q} a_{2 q}=-\sum_{s=0}^{s=q q-1}(-1)^{s}\left(\begin{array}{c}
r+2 q \\
r+s
\end{array}\right) 2^{s} m^{2 x-s} a_{s} \\
& (-1)^{q}\left(b_{2_{q}}-2^{2 q} a_{2 q}\right)=\sum_{s=0}^{s=q-1}(-1)^{s}\left(\begin{array}{l}
r+2 q \\
r+2 s
\end{array}\right) 2^{2 s} m^{2 q-2 s} a_{2 s} E_{q-s} \\
& (-1)^{q}\left(b_{2 q}-2^{2 q} a_{2 q}\right)=\sum_{s=0}^{s=q-1}(-1)^{s}\left(\begin{array}{c}
r+2 q \\
r+2 s+1
\end{array}\right)\left(\frac{m}{2}\right)^{2 q-2 s-1} 2^{2 s+1} a_{2 s+1} T_{q-s} .
\end{aligned}
$$


Ici nous aurons le théorème suivant:

II. Soient tous les $\alpha_{s}$ el tous les $\beta_{s}$ des nombres entiers, les quantités $n$, $a_{q}$ et $b_{\mathrm{s}_{q}}$ ont la même propriété, et nous aurons les deux congruences

$$
\begin{gathered}
2 a_{2_{q+1}} \equiv 0(\bmod m), \quad q \geqq 0 \\
b_{2 q} \equiv 2^{2 q} a_{2 q}\left(\bmod m^{2}\right), \quad q \geqq 1 .
\end{gathered}
$$

En effet, il est évident que $m$ et $a_{s}$ sont des nombres entiers, et la formule (19) montre que $b_{2_{2}}$ est également un entier; les deux congruences en question sont ensuite des conséquences immédiates de (12) et (20).

Les formules précédentes donnent des résultats remarquables concernant les zéros d'un polynome régulier quelconque.

Posons $m=1, \beta_{s}=1$, ce qui donnera $\varepsilon=r=0$; posons ensuite $\alpha_{s}=-\omega_{s}$, les $n$ nombres $\omega_{s}$ sont les zéros d'un polynome régulier quelconque du degré $n$. En nous bornant à des sommes de puissances semblables des zéros

$$
s_{q}=\omega_{1}^{q}+\omega_{2}^{q}+\cdots \omega_{n}^{q}, \quad s_{0}=n,
$$

nous avons à posel dans les formules précédentes

$$
a_{r}=(-1)^{r} s_{r},
$$

ce qui donnera le théorème suivant:

III. Les sommes des puissances semblables des zéros d'un polynome régulier quelconque satisfont à des conditions analogues à celles que nous venons de développer pour les coefficients du polynome susdit.

Dans les paragraphes suivants nous avons à combiner des hypothèses simples relatives aux nombres $\beta_{s}$ et les hypothèses relatives aux $\alpha_{s}$ étudiées dans le paragraphe III, ce qui nous donnera un grand nombre de formules analogues.

Dans un Mémoire récent intitulé: Verkürzte Rekursionsformeln für die Bernoullischen und Eulerschen Zahlen (*) j'ai étudié des cas particuliers de nos polynomes $\Phi_{q}(x)$; ces cas sont intimément liés avec les recherches de KRONEGKER (**) et ils nous donnent particulièrement les formules très remarquables indiquées par M. Haussner (**) pour les $B_{n}, T_{n}$ et $E_{n}$ et une suite de formules analogues.

(*) Sitzungsberichte der Kgl. sächsischen Gesellschaft der Wissenschaften zu Leipzig, 1913. (**) Journal de Mathématiques pures et appliquées (2), t. I, pp. 385-391; 1856. (**) Göttinger Nachrichten 1893, pp. 777-809. 


\section{Première application.}

Posons, comme dans le théorème III du paragraphe IV,

$$
\beta_{s}=1,
$$

ce qui donnera $\hat{\varepsilon}=r=0$, nous aurons à considérer ces trois cas partieuliers :

Premier cas particulier. L'ensemble des $\alpha_{s}$ étant

$$
1,2,3, \ldots, n-1 \text {, }
$$

nous avons à remplacer, dans les formules générales du paragraphe III, $n$ par $n-1$ et à poser $m=n$ et

$$
a_{g}=s_{q}(n-1), \quad a_{0}=n-1,
$$

ce qui donnera la congruence

$$
2 s_{2 q+1}(n-1) \equiv 0(\bmod n) .
$$

Quant au nombre $b_{2 q}$, nous aurons selon que $n=2 u$ ou $n=2 \mu+1$ respectivement

$$
b_{2 q}=2^{2 \mu+1} s_{2 q}(\mu-1), \quad b_{2 q}=2 t_{2 q}\left({ }^{\prime \prime}\right),
$$

d'où en remplaçant $\mu$ par $n$

$$
\left.\begin{array}{l}
2^{2 q+1} s_{2_{q}}(n-1) \equiv 2^{2 q} s_{2_{Q}}(2 n-1)\left(\bmod 4 n^{2}\right) \\
t_{2_{q}}(n) \equiv 2^{2 q-1} s_{2_{q}}(2 n)\left(\bmod (2 n+1)^{2}\right) .
\end{array}\right\}
$$

Soit au contraire l'ensemble des $\alpha_{s}$ les $n+1$ nombres

$$
0,1,2,3, \ldots, n
$$

nous aurons au lieu de (2) les expressions suivantes

$$
a_{u}=s_{q}(n), \quad a_{0}=n+1,
$$


ce qui donnera la formule d'Euler (*)

$$
\left(1-(-1)^{4}\right) s_{q}(n)=(n+1) n^{t}+\sum_{r=1}^{r=q-1}(-1)^{r}\left(\begin{array}{l}
q \\
r
\end{array}\right) n^{q-r} s_{r}(n),
$$

dans laquelle il est permis de remplacer $s_{\nu}(n)$ par $s_{\nu}(n-1)$, pourvu que le premier terme du second membre soit remplacé par $(n-1) n^{q}$.

Comme autre cas particulier connu nous aurons

$$
\left.\begin{array}{rl}
(-1)^{q}\left(s_{2 q+1}(n-1)\right. & \left.-n\left(q+\frac{1}{2}\right) s_{2 q}(n-1)\right)= \\
= & \sum_{r=0}^{r=q-1}(-1)^{r}\left(\begin{array}{l}
2 q+1 \\
2 r+1
\end{array}\right) n^{2 q-2 r} s_{2 r+1}(n-1) B_{q-r},
\end{array}\right\}
$$

formule qui a été trouvée presque contemporainément par A. RADIcke (**) et J. WonpITZKY (***); posons, dans (8), $n=2$, nous retrouvons la formule d'EULER $\left.{ }^{* * * *}\right)$

$$
\sum_{r=0}^{q-}(-1)^{r}\left(\begin{array}{l}
2 q+1 \\
2 r+1
\end{array}\right) 2^{2 q-2 r} B_{q-r}=(-1)^{q-1} 2 q
$$

Appliquons au contraire la seconde hypothèse, puis soustrayons (8) et la formule analogue ainsi obtenue, nous aurons la première formule récursive connue pour les nombres de Bennoulin, savoir la formule de Morvre $\left({ }_{*}^{*}\right)$

$$
(-1)^{q-1}\left(q-\frac{1}{2}\right)=\sum_{r=0}^{=\prime-1}(-1)^{r}\left(\begin{array}{l}
2 q+1 \\
2 r+1
\end{array}\right) B_{q-r}
$$

Deuxième cas particulier. Soit l'ensemble des $\alpha_{s}$ les nombres impairs

$$
1,3,5, \ldots, 2 n-1
$$

nous aurons à poser dans les formules générales $m=2 n$ et

$$
a_{q}=t_{q}(n), \quad a_{0}=n,
$$

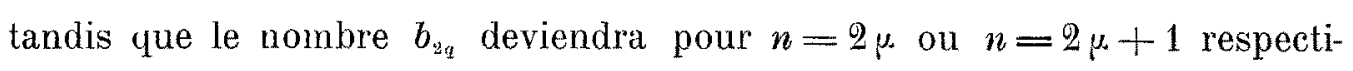

(*) Tnstitutiones calculi differentialis, pp. 348-351. Saint-Pétersbourg, 1755.

(**) Die Recursionsformeln für die Bernoullischen und Eulerschen Zahlen, p. 7; Halle, 1880.

(*****) Joumal de Crelle, t. 84, p. 217; 1883.

(*****) Opuscula analytica, t. II, pp. 264-265. Saint-Pétersbourg, 1785.

$\left({ }_{*}^{*}\right)$ Miscellanea analytica de seriebus et quadraturis. Londres, 1730. 
vement

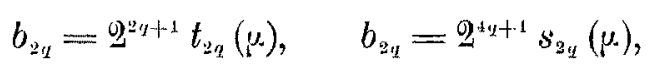

(le sorte que nous aurons les congruences

$$
\begin{aligned}
t_{2 q+1}(n) & \equiv 0(\bmod n) \\
2^{2 q+1} t_{2 q}(n) & \equiv 2^{24} t_{2_{q}}(2 n)\left(\bmod 4 n^{2}\right) \\
2^{2 x+1} s_{2 q}(n) & \equiv t_{2 q}(2 n+1)\left(\bmod (2 n+1)^{2}\right) .
\end{aligned}
$$

Troisième cas particulier. Soient les $\alpha_{s}$ les positits entiers plus petits (fue $m$ et premiers avec $m$, nous aurons

$$
2 s_{2_{1+1}} \equiv 0(\bmod m)
$$

et, pourvu que $m$ soit un nombre impair,

$$
t_{2_{q}} \equiv 2^{2_{q-1}} s_{q_{q}}\left(\bmod m^{2}\right) \text {. }
$$

L'analogie des trois cas particuliers que nous venons d'étudier est parfaite.

\section{Dhuxième application.}

En second lieu nous posons

$$
\beta_{s}=(-1)^{n-s},
$$

ce qui donnera $\varepsilon=0$ ou $\varepsilon=1$, selon que $n$ est impair ou pair.

I. Soit $n$ un nombre impair, nous aurons $\varepsilon=r=0$, ce qui donnera des formules analogues à celles du paragraphe $\mathrm{V}$.

Premier cas particulier. Pour les nombres naturels

nous trouvons

$$
1,2,3,4, \ldots, 2 n-1
$$

$$
m=2 n, \quad a_{q}=\sigma_{q}(2 n-1), \quad b_{2 q}=2^{2 q+1} \sigma_{2 q}(n-1),
$$

ce qui donnera

$$
\left.\begin{array}{rl}
\sigma_{2 q+1}(2 n-1) & \equiv 0(\bmod u) \\
2^{2++1} \sigma_{2_{q}}(n-1) & \equiv 2^{2 q} \sigma_{2 q}(2 n-1)\left(\bmod 4 u^{2}\right) .
\end{array}\right\}
$$


Second cas particulier. Pour les nombres impairs

nous avons à poser

$$
1,3,5, \ldots, 4 n+1
$$

$$
m=4 n+2, \quad a_{q}=\tau_{q}(2 n+1), \quad b_{2 q}=2^{4 q+1} \sigma_{2 q}(n),
$$

de sorte que nous aurons

$$
\left.\begin{array}{rl}
\tau_{2 q+1}(2 n+1) & \equiv 0(\bmod (2 n+1)) \\
2^{2 x+1} \sigma_{2 q}(n) & \equiv \tau_{2_{q}}(2 n+1)\left(\bmod (2 n+1)^{2}\right) .
\end{array}\right\}
$$

II. Soit $n$ un nombre pair, nous aurons $\varepsilon=1$, tandis que nous ne pouvons dire généralement que $r$ doit être un nombre impair.

Dans nos trois cas ordinaires nous aurons toujour's $r=1$.

Premier cas particulier. L'ensemble des nombres $\alpha_{s}$

donnera

$$
1,2,3,4, \ldots, 2 n
$$

$$
m=2 n+1, \quad a_{q}=\sigma_{q+1}(2 n), \quad b_{a_{q}}=\tau_{2 q+1}(n),
$$

et nous aurons par conséquent

$$
\left.\begin{array}{rl}
\sigma_{2 u+2}(2 n) & \equiv 0(\bmod (2 n+1)) \\
\tau_{2 y+1}(n) & \equiv 2^{3_{4}} \sigma_{2_{u+1}}(2 n)\left(\bmod (2 n+1)^{2}\right) \cdot
\end{array}\right\}
$$

Deuxième cas particulier. Pour l'ensemble des nombres impairs

$$
1,3,5,7, \ldots, 4 n-1
$$

nous aurons

$$
m=4 n, \quad a_{4}=\tau_{2_{q+1}+1}(2 n), \quad b_{2_{4}}=2^{2 q+1} \tau_{2_{2+1}}(n),
$$

de sorte que nous aurons

$$
\left.\begin{array}{rl}
2 \tau_{3 q+2}(2 n) & \equiv 0(\bmod 4 n) \\
2^{2 y+1} \tau_{2 q+1}(n) & \equiv 2^{24} \tau_{2 x+1}(2 n)\left(\bmod 16 n^{2}\right) .
\end{array}\right\}
$$

Troisième cas particulier. Soit $m$ un nombre impair et soient les $\alpha_{s}$ les 
nombres plus petits que $m$ et premiers avec $m$, nous aurons

$$
\left.\begin{array}{rl}
\sigma_{2 q+2} & \equiv 0(\bmod m) \\
\tau_{2 q+1} & \equiv 2^{2 q} \sigma_{2_{q+1}}\left(\bmod m^{2}\right) .
\end{array}\right\}
$$

L'analogie de ces formules entre elles et avec celles du paragraphe précédent est parfaite.

Additionnons, puis soustrayons les formules récursives obtenues dans ces deux paragraphes, nous aurons de nombreuses d'autres formules de ce genre. Cependant nous ne nous arrêtons pas à une étude plus ample de ce problème.

\section{Trotsième application.}

Pour donner d'autres applications de nos formules générales nous remplaçons $n$ par $n+1$ et nous nous bornerons à l'étude de ces deux cas particuliers de l'ensemble des $\alpha_{s}$ :

$$
\begin{aligned}
& 0,1,2,3, \ldots, n, \quad m=n \\
& 1,3,5,7, \ldots, 2 n+1, \quad m=2 n+2 .
\end{aligned}
$$

En premier lieu posons

$$
\psi_{s}=\left(\begin{array}{c}
n \\
s
\end{array}\right)
$$

nous aurons $\varepsilon=r=0$; posons ensuite pour abréger

$$
\begin{aligned}
& a_{n, q}=\sum_{s=0}^{s=n-1}\left(\begin{array}{l}
n \\
s
\end{array}\right)(n-s)^{q} \\
& b_{n, q}=\sum_{s=0}^{\sum n-1}\left(\begin{array}{l}
n \\
s
\end{array}\right)(n-2 s)^{t} \\
& c_{n, q}=\sum_{s=0}^{s=n}\left(\begin{array}{l}
n \\
s
\end{array}\right)(2 n-2 s+1)^{q},
\end{aligned}
$$

l'hypothèse (1) donnera

$$
a=a_{n, \Psi}, \quad b_{2 q}=2 b_{\eta_{1,4},}
$$


de sorte que nous aurons les congruences

$$
\left.\begin{array}{rl}
2 a_{n, 2 q+1} & \equiv 0(\bmod n) \\
2 b_{n, q_{q}} & \equiv 2^{2 q} a_{n, u_{q}}\left(\bmod n^{2}\right) .
\end{array}\right\}
$$

Quant à l'ensemble (2), nous aurons

d'où l'on conclut

$$
\left.\begin{array}{rl}
a_{q} & =c_{n, q}, \quad b_{2 q}=2^{2 q+1} b_{n, 2_{q}}, \\
c_{n, 2 q+1} & \equiv 0(\bmod (n+1)) \\
2^{2 q} b_{n, 2_{q}} & \equiv 2^{2 q-1} c_{n, 2_{q}}\left(\bmod (n+1)^{q}\right) .
\end{array}\right\}
$$

En second lieu nous posons

$$
\beta_{s}=(-1)^{n-s}\left(\begin{array}{l}
n \\
s
\end{array}\right)
$$

ce qui donnera $\varepsilon=r=n$; dans ce cas nous posons

$$
\begin{aligned}
& \alpha_{n, q}=\sum_{s=0}^{s=n-1}(-1)^{s}\left(\begin{array}{l}
n \\
s
\end{array}\right)(n-s)^{q+n} \\
& \beta_{n, q}=\sum_{s=0}^{\sum \frac{n-1}{2}}(-1)^{s}\left(\begin{array}{c}
n \\
s
\end{array}\right)(n-2 s)^{q+n} \\
& \gamma_{n, q}=\sum_{s=0}^{s=n}(-1)^{s}\left(\begin{array}{c}
n \\
s
\end{array}\right)(2 n-2 s+1)^{q+n} .
\end{aligned}
$$

Cela posé, nous aurons pour l'ensemble (1)

$$
a_{q}=x_{n, q}, \quad b_{a_{q}}=\frac{(-1)^{n}}{2^{n-1}} \beta_{n, 2 q}
$$

c'est-à-dire que $\beta_{n, 2 q}$ est toujours divisible par $2^{2-1}$; dans ce cas nous aurons les congruences

$$
\left.\begin{array}{c}
2 \alpha_{n, 2, q+1} \equiv 0(\bmod n) \\
\frac{(-1)^{n}}{2^{n-1}} \beta_{n, 2 q} \equiv 2^{2 q} \alpha_{n, 2 q}\left(\bmod n^{2}\right) .
\end{array}\right\}
$$

Quant à l'ensemble (2), nous aurons

$$
a_{q}=\gamma_{n, q}, \quad b_{2 q}=\frac{(-1)^{n} 2^{2 q}}{2^{n-1}} \beta_{n, 2 q}
$$


ce qui domnera

$$
\left.\begin{array}{c}
\gamma_{n, 2, q+1} \equiv 0(\bmod (n+1)) \\
(-1)^{n}{\frac{2}{2 q} \beta_{n, 2 q u}}_{2^{n-1}} \equiv 2^{2 q} \gamma_{n, 2 q}\left(\bmod (2 n+2)^{2}\right) .
\end{array}\right\}
$$

On sait que les six nombres $\alpha_{n, q}$ etc. que nous venons de considérer jouent un rôle important dans les représentations indépendantes des nombres $B_{n}, T_{n}$ et $E_{n}$.

\section{LES FONCTIONS PARTIFLLES.}

Revenons aux ensembles contenant les $n$ éléments $z_{s}$ et les $n$ éléments correspondants $\beta_{s}$ que nous avons appliqués dans le paragraphe $I V$, puis supposons que $n$ soit un nombre pair, savoir $n=2$, , il est possible de diviser en deux groupes à $\mu$. éléments les nombres $\alpha_{s}$, savoir

$$
\begin{aligned}
& \alpha_{1}^{\prime} \alpha_{2}^{\prime} \alpha_{3}^{\prime} \ldots \alpha_{\beta}^{\prime} \\
& \left.\alpha^{\prime \prime}{ }_{1} \alpha^{\prime \prime}{ }_{2} \alpha^{\prime \prime}{ }_{3} \ldots \alpha^{\prime \prime}{ }_{\mu},\right\}
\end{aligned}
$$

de sorte que nous aurons pour $1 \leqq s \leqq \mu$

$$
\alpha_{s}^{\prime}+\alpha_{s}^{\prime \prime}=m,
$$

et ce problème admet précisément $2^{\mu-1}$ solutions.

Nous désignons comme complémentaires les deux groupes (1) ainsi définies, tandis qu'mn seul de ces deux groupes complémentaires est un groupe partiel formé de l'ensemble des $n$ nombres $\alpha_{s}$.

Nous divisons aussi en deux groupes les nombres $\beta_{s}$ correspondantes $\grave{a}(1)$, de sorte que $\xi^{\prime}{ }_{s}$ et $\beta^{\prime \prime}{ }_{s}$ appartiennent à $\alpha_{*}^{\prime}$ respectivement $\alpha_{*}^{\prime \prime}$; c'est-à-dire que nous aurons pour $1 \leqq s \leqq \mu$

$$
\beta^{\prime}=(-1)^{*} \beta_{s}^{\prime \prime} .
$$

Soient maintenant (1) deux groupes complémentaires formés de l'ensemble des $n$ nombres $\alpha_{*}$, nous désignons comme fonctions partielles appartenant aux ensembles des nombres $\alpha_{s}$ et des nombres $\beta_{s}$ chacun des deux 
polynomes entiers

$$
\left.\begin{array}{l}
f_{v}(x)=\frac{1}{y !} \cdot \sum_{s=1}^{s=\mu} \beta_{*}^{\prime}\left(x+\frac{x_{s}^{\prime}}{m}\right)^{\nu} \\
g(x)=\frac{1}{y !} \cdot \sum_{s=1}^{s=\mu} \beta_{*}^{\prime \prime}\left(x+\frac{\alpha_{s}^{\prime \prime}}{m}\right)^{\nu},
\end{array}\right\}
$$

où $\nu \geqq 0$ est un entier quelconque du reste.

Nous désignons comme complémentaires les deux fonctions partielles ainsi définies $f_{v}(x)$ et $g_{v}(x)$.

Ces définitions adoptées, nous pouvons former précisément $2^{\mu}$ fonctions partielles qui correspondent aux ensembles des $\alpha_{s}$ et des $\beta_{s}$.

Supposons maintenant $n$ impair, savoir $n=2 \mu+1$, nous a vons à ajoindie à chacun dess deux groupes complémentaires (1) l'élément

$$
\frac{m}{2}
$$

et les fonctions partielles complémentaires $f,(x)$ et $g \nu(x)$ deviennent dans ce ras

$$
\left.\begin{array}{l}
f_{\nu}(x)=\frac{1}{v !}\left(\frac{\beta_{\mu+1}}{2}\left(x+\frac{1}{2}\right)^{\nu}+\sum_{s=1}^{s=\mu} \varepsilon_{*}^{\prime}\left(x+\frac{\alpha_{s}^{\prime}}{m}\right)^{\nu}\right) \\
g_{\nu}(x)=\frac{1}{v !}\left(\frac{\beta_{\mu+1}}{2}\left(x+\frac{1}{2}\right)^{\nu}+\sum_{s=1}^{s=\mu} \beta_{*}^{\prime \prime}\left(x+\frac{\alpha_{s}^{\prime \prime}}{m}\right)^{\nu}\right)
\end{array}\right\}
$$

Dans ces deux définitions nous supposons $n>1$; soit particulièrement $n=1$, chacun des deux groupes complémentaires (1) est composé du seul élément (4), de sorte que nous aurons dans ce cas seulement les deux fonctions partielles complémentaires

$$
f \cdot(x)=y(x)=\frac{\beta_{1}}{v ! 2}\left(x+\frac{1}{2}\right)^{\prime} .
$$

Cela posé, la définition (6) du paragraphe IV donnera le théorème suivant:

I. Soient $f_{\nu}(x)$ et $g_{\nu}(x)$ deux fonctions partielles complémentaires quelconques appartenant aux ensembles des $\alpha_{*}$ et des $\beta_{s}$, nous aurons toujours

$$
\begin{gathered}
f_{\nu}(x)+g_{\nu}(x)=\Phi_{\nu-r}(x), \quad \nu \geqq r \\
(-1)^{r+s} f_{\nu}(-x-1)=g_{\nu}(x) . \quad(-1)^{r+\varepsilon} g_{\nu}(-x-1)=f_{\nu}(x) .
\end{gathered}
$$


Posons ensuite pour abréger

$$
\left.\begin{array}{rl}
f_{\nu}(x) & =\sum_{s=0}^{s=\nu} \frac{a_{s}^{\prime}}{s ! m^{s}} \cdot \frac{x^{\gamma-s}}{(v-s) !} \\
g_{\nu}(x) & =\sum_{s=0}^{s=y} \frac{a_{s}^{\prime \prime}}{s ! m^{s}} \cdot \frac{x^{y-s}}{(v-s) !},
\end{array}\right\}
$$

nous aurons, en vertu du développement (8) du paragraphe IV,

$$
a_{s}^{\prime}+a_{s}^{\prime \prime}=a_{s-r}, \quad s \geqq r
$$

supposons $s<r$, nous aurons, au contraire,

$$
a_{s}^{\prime}+a_{s}^{\prime \prime}=0 \text {. }
$$

Dans ce cas il faut supposer naturellement $r>0$.

Les fonctions partielles jouent, pour nos recherches ultérieures, un rôle fondamental.

\section{TROISIÈME PARTIE.}

\section{Applications des fonctions $B_{n}(p x)$ et $E_{n}(p x)$.}

\section{Formulfs Fondamentales.}

Soit $p$ un positif entier quelconque, les équations aux différences finies qui figurent dans les définitions des fonctions de Bernoulu et d'Eulrir, savoir les formules (1) et (:3) du paragraphe I, donnent immédiatement ces deux identités générales

$$
\begin{gathered}
B_{n}(x-p)=B_{n}(x)-\frac{1}{(n-1) !} \cdot \sum_{s=0}^{s=p-1}(x-s)^{n-1} \\
E_{n}(x-p)=(-1)^{p} E_{n}(x)-\frac{(--1)^{p}}{n !} \cdot \sum_{s=0}^{s=p-1}(-1)^{s}(x-s)^{n},
\end{gathered}
$$


formules qui nous conduiront à des résultats essentiels pour les deux fonctions

$$
\varphi(x)=\frac{B_{n}(p x-\beta)}{p^{n-1}}, \quad \psi(x)=\frac{E_{n}(p x-\beta)}{p^{n}}
$$

où $\beta$ désigne un nombre complexe quelconque.

En effet, substituons $-x-1$ au lieu de $x$, nous aurons en vertu de (1) et (2)

$$
\begin{gathered}
\varphi(-x-1)=\frac{B_{n}(-p x-\beta)}{p^{n-1}}+\frac{(-1)^{n}}{(n-1) !} \cdot \sum_{s=0}^{s=p-1}\left(x+\frac{\beta+s}{p}\right)^{n-1} \\
\psi(-x-1)=\frac{(-1)^{p} E_{n}(-p x-\beta)}{p^{n}}-\frac{(-1)^{n+p}}{n !} \cdot \sum_{s=0}^{s=p-1}(-1)^{s}\left(x+\frac{\beta+s}{p}\right)^{n}
\end{gathered}
$$

d'où, en appliquant les équations fonctionnelles (6) du paragraphe I,

$$
\left.\begin{array}{c}
(-1)^{n} \varphi(-x-1)=\frac{B_{n}(p x+\beta-1)}{p^{n-1}}+\frac{1}{(n-1) !} \cdot \sum_{s=0}^{s=p-1}\left(x+\frac{\beta+s}{p}\right)^{n-1} \\
(-1)^{n} \psi(-x-1)=\frac{(-1)^{p} E_{n}(p x+\beta-1)}{p^{n}}+ \\
+\frac{1}{n !} \cdot \sum_{s=0}^{s=p-1}(-1)^{p-s-1}\left(x+\frac{\beta+s}{p}\right)^{n}
\end{array}\right\}
$$

formules qui nous conduiront à des résultats intéressants si nous supposons $\beta=0$ ou $\beta=\frac{1}{2}$.

Posons d'abord $\beta=0$, puis appliquons encore une fois les deux équations aux différences finies qui figurent dans la définition des $B_{n}(x)$ et des $E_{n}(x)$, nous aurons pour les fonctions

$$
\varphi(x)=\frac{B_{n}(p x)}{p^{n-1}}, \quad \psi(x)=\frac{E_{n}(p x)}{p^{n}}
$$

les équations fonctionnelles

$$
\left.\begin{array}{l}
(-1)^{n} \varphi(-x-1)=\varphi(x)+\frac{1}{(n-1) !} \cdot \sum_{s=1}^{s=p-1}\left(x+\frac{s}{p}\right)^{n-1} \\
(-1)^{n+p-1} \psi(-x-1)=\psi(x)+\frac{1}{n !} \cdot \sum_{s=1}^{s=p-1}(-1)^{s}\left(x+\frac{s}{p}\right)^{n} .
\end{array}\right\}
$$


Posons ensuite $\beta=\frac{1}{9}$, nous verrons que les fonctions

$$
\Psi_{1}(x)=\frac{B_{n}\left(p x-\frac{1}{2}\right)}{p^{n-1}}, \quad \psi_{1}(x)=\frac{E_{n}\left(p x-\frac{1}{2}\right)}{p^{n}}
$$

satisfont aux équations fonctionnelles

$$
\begin{array}{ll}
(-1)^{n} p_{1}(-x-1)=\psi_{1}(x)+\frac{1}{(n-1) !} \sum_{s=0}^{s=p-1}\left(x+\frac{2 s+1}{2 p}\right)^{n-1} & \mid \\
(-1)^{n+p} \psi_{1}(-x-1)=\psi_{1}(x)-\frac{1}{n !} \cdot \sum_{s=0}^{s=p-1}(-1)^{s}\left(x+\frac{2 s+1}{2 p}\right)^{n} \cdot & \mid
\end{array}
$$

Or, il est très facile de généraliser beaucọup les deux dernières groupes d'équations fonctionnelles.

A cet effet, considérons le nombre composé $m$ étudiè à la fin du paragraphe III, puis posons, en appliquant les définitions susdites,

$$
I_{n}^{n}(x)=\frac{B_{n}(m x)}{m^{n-1}}+\sum_{x=1}^{s=r}(-1)\left(\sum_{q=1}^{q=t_{s}} \frac{B_{n}\left(m_{q}^{(s)} x\right)}{\left(m_{q}^{(s)}\right)^{n-1}}\right)
$$

la formule (26) du \$ ILI montrera, en vertu de la première relation (2), que la fonction $F_{n}^{*}(x)$ satisfait à l'équation fonctionnelle

$$
(-1)^{\prime \prime} F_{n}(-x-1)=F_{n}(x)+\frac{1}{(u-1) !} \cdot \sum_{s=1}^{s=2 \mu}\left(x+\frac{a_{s}}{m}\right)^{n-1}, \quad u \geqq 1,
$$

où $\mathcal{Q}_{\mu}=p(m)$, et où les $\alpha_{1} a_{2} a_{3} \ldots a_{2 \mu}$ sont les positifs entiers plus petits que $n$ et premier's avec $n$.

Supposons ensulte que $m$ soit un nombre impair, puis posons

$$
G_{n}(x)=\frac{E_{n}^{\prime}(m x)}{m^{n}}+\sum_{s=1}^{s=r}(-1)\left(\sum_{q=1}^{q=t_{s}} \frac{E_{n}\left(m_{q}^{(s)} x\right)}{\left(m_{q}^{(s)}\right)^{n}}\right)
$$

nous aturons de même

$$
(-1)^{n} G_{n}(-x-1)=G_{n}(x)+\frac{1}{n !} \cdot \sum_{s=1}^{s=z_{n}^{\prime}}(-1)^{\alpha}\left(x+\frac{a_{s}}{m}\right)^{n}
$$

Il est très facile d'ordonner selon des puissances descendantes de $x$ les deux polynomes $F_{n}(x)$ et $G_{n}(x)$.

En effet, désignons par a un positif entier, puis posons pour abréger

$$
q_{1 \alpha}(m)=\left(p_{1}^{a}-1\right)\left(p_{s}^{a}-1\right) \ldots\left(p_{r}^{a}-1\right),
$$


nous aurons les expressions suivantes

$$
\begin{aligned}
& F_{n}(x)=\frac{\varphi_{1}(m)}{n !} x^{n}+\sum_{s=1} \frac{n}{2} \frac{(-1)^{n+s-1}}{(2 s) ! m^{2 s-1}}(m) B_{s} \cdot \frac{x^{n-2 s}}{(n-2) !} \\
& G_{n}(x)=\frac{1}{2} \sum_{s=1}^{\leq \frac{n+1}{2}} \frac{(-1)^{r+x-1} \varphi_{s s-1}(m) T_{s}}{(2 s-1) !(2 m)^{2 s-1}} \cdot \frac{x^{n-2 s+1}}{(n-2 s+1) !},
\end{aligned}
$$

où il faut admettre $n \geqq 2$ respectivement $n \geqq 1$; supposons dans $(10) n=1$, le second membre doit être réduit ì son premier terme.

Nous ne nous arrêtons pas à la généralisation correspondante des formules (7), parce qu'une telle généralisation nous ne donne pas des applications aussi intéressantes que la précédente.

\section{SUITES RÉGuLit̀res DE PREMLÈre ESPÈCE.}

Désignons par $f_{m}(x)$ et $g_{n}(x)$ deux fonctions partielles complémentaires quelconques, formées de l'ensemble

$$
1,2,3, \ldots, p-1
$$

des nombres $\alpha_{*}$ et de l'ensemble adjoint des nombres $\beta_{*}$

puis posolls

$$
\psi_{s}=1
$$

$$
f_{m}(x)=\sum_{q=0}^{q=m} \frac{s^{\prime}}{q !} \cdot \frac{x^{m-q}}{(m-q) !}, \quad g_{n+}(x)=\sum_{q=0}^{q=m} \frac{s^{\prime \prime}}{q ! p^{4}} \cdot \frac{x^{m-4}}{(m-q) !},
$$

nous aurons, en vertu des formules (10) et (11) du paragraphe VIII,

$$
s_{i t}^{\prime}+s_{{ }_{4}}^{\prime \prime}=s_{i t}(p-1), \quad s_{0}^{\prime}=s^{\prime \prime}{ }_{0}=\frac{p-1}{2} .
$$

Dans ce qui suit nous désignons comme complémentaires les deux sommes aiusi définies $s_{q}^{\prime}$ et $s^{\prime \prime}{ }_{q}$.

Soit $p=2 r+1$ ou $p=2 r+2$, nous pouvons former des ensembles (1) et (2) précisément $2^{\prime \prime}$ fonctions partielles. 
Cela posé, nous aurons le théorème suivant:

I. Soit $f_{n}(x)$ une fonction partielle quelconque formée des ensembles (1) et (2), les fonctions

$$
H_{n}(x)=\frac{B_{n}(p x)}{p^{n-1}}+f_{n-1}(x), \quad n \geqq 1,
$$

sont toutes des polynomes réguliers.

En effet, nous aurons immédiatement $F^{\prime \prime}{ }_{n}(x)=F_{n-1}(x)$ et les formules (8) du paragraphe VIII et (6) du paragraphe IX donnent

$$
(-1)^{\prime \prime} F_{n}(-x-1)=\frac{B_{n}(p x)}{p^{n-1}}+\left(f_{n-1}(x)+g_{n-1}(x)\right)-g_{n-1}(x)=F_{n}(x) .
$$

Quant au développement du polynome $F_{n}(x)$ selon les fonctions de Bernoulu, nous ayous à chercher, dans $F_{n}(x)$, les coefficients des puissances $x^{n-2 x-1}$. Or, $B_{u}(x)$ ne contenant qu'une seule de ces puissances, savoir $x^{n-1}$, les autres se trouvent dans la fonction partielle $f_{n-1}(x)$.

Appliquons ensuite les formules (3) et (4), nous aurons le développement cherché

$$
\frac{1}{2} F_{n}(x)=\frac{p}{2} B_{n}(x)+\sum_{q=1}^{\leqq \frac{n}{2}} \frac{s_{2 q}^{\prime}}{(2 q) ! p^{2 q}} B_{n-2 q}(x),
$$

et il existent par conséquent précisément 2" développements de ce genre, ce qui nous donnera des formules récursives très remarquables.

En effet, substituons, dans (6), $2 n$ au lieu de $n$, puis posons $x=0$, nous aurons

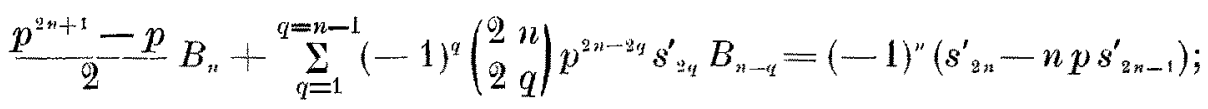

nous nous bornons à cette seule application de la formule générale (6).

Introduisons maintenant, dans (2), au lieu des $s^{\prime}{ }_{2}$ les sommes complémentaires $s^{\prime \prime}{ }_{n}$, ce qui est permis, puis additionnons les deux formules ainsi obtenues, nous aurons, quelles que soient les fonctions partielles complémentaires $f_{n}(x)$ et $g_{m}(x)$ que nous avons prises pour point de départ:

$$
\begin{gathered}
p\left(p^{2 n}-1\right) B_{n}+\sum_{q=1}^{q=n-1}(-1)^{q}\left(\begin{array}{ll}
2 & n \\
2 & q
\end{array}\right) p^{2 n-2 n} s_{2 q}(p-1) B_{n-q}= \\
=(-1)^{n}\left(s_{2 n}(p-1)-n p s_{z n-1}(p-1)\right)
\end{gathered}
$$


ce qui est précisément la seconde formule de ce genre trourée par A. RA$\operatorname{DICKE}\left(^{*}\right)$ et J. WoRPITZKY $\left(^{* *}\right)$.

Posons dans (8) $p=2$, nous aurons, en vertu de la formule eulérienne (13) du paragraphe 1 ,

$$
\frac{n T_{n}}{2^{2 n-2}}=\sum_{q=1}^{q=n-1}(-1)^{q-1}\left(\begin{array}{l}
2 n \\
2 q
\end{array}\right) 2^{2 n-2 q} B_{n-q}+(-1)^{n-1}(2 n-1)
$$

posons dans (7)

$$
p=3, \quad s_{m}^{\prime}=1, \quad s_{m}^{\prime \prime}=2^{m}
$$

nous aurons ces deux autres formules récursives d'une simple forme

$$
\begin{gathered}
3^{2 n+1}-3 B_{n}+\sum_{q=1}^{q=n-1}(-1)^{q}\left(\begin{array}{l}
2 n \\
2 q
\end{array}\right) 3^{2 n-2 q} B_{n-q}=(-1)^{n-1}(3 n-1) \\
\frac{3^{2 n+1}-3}{2} B_{n}+\sum_{q=1}^{q=n-1}(-1)^{q}\left(\begin{array}{l}
2 n \\
2 q
\end{array}\right) 2^{2 q} 3^{2 n-2 q} B_{n-q}=(-1)^{n-1}(3 n-2)^{2} 2^{2 n-1} .
\end{gathered}
$$

Soit maintenant l'ensemble adjoint à (1) défini par

$$
\beta_{s}=(-1)^{s} \text {, }
$$

nous formons deux fonctions partielles complémentaires $h_{m}(x)$ et $k_{m}(x)$ de ces deux ensembles. Posons ensuite

$$
h_{i n}(x)=\sum_{q=0}^{q=m} \frac{\sigma_{q}^{\prime}}{q ! p^{q}} \cdot \frac{x^{m-q}}{(m-q) !}, \quad k_{m}(x)=\sum_{q=0}^{q=m} \frac{\sigma^{\prime \prime}}{q ! p^{q}} \cdot \frac{x^{m-q}}{(m-q) !},
$$

les sommes complémentaires $\sigma^{\prime}{ }_{q}$ et $\sigma^{\prime \prime}{ }_{2}$ satisfont à la condition

$$
\sigma_{q}^{\prime}+\sigma^{\prime \prime}{ }_{q}=(-1)^{p-1} \sigma_{q}(p-1)
$$

ce qui domnera pour $q=0$

$$
\sigma_{0}^{\prime}+\sigma_{0}^{\prime \prime}=0, \quad \sigma_{0}^{\prime}+\sigma_{0}^{\prime \prime}=-1
$$

selon que $p$ est supposé impair ou pair.

Cela posé, nous trouvons le théorème suivant:

II. Soit $h_{n}(x)$ une fonction partielle quelconque formée des ensembles

(*) Die Recursionsformeln für die Bernoullischen und Eulerschen Zahlen, p. 7. Halle, 1880 . (**) Jownal de Crelle, t. 84, p. 217; 1883 . 
(1) et (12), les 2 fonctions de la forme

$$
G_{n}(x)=E_{n} \frac{(p x)}{p^{n}}+h_{n}(x)
$$

sont toutes des polynomes réguliers.

Nous aurons dans ce cas

$$
(-1)^{n+y-1} G_{n}(-x-1)=\frac{E_{n}(p x)}{p^{n}}+\left(h_{n}(x)+k_{n}(x)\right)-k_{n}(x)=G_{n}(x)
$$

c'est-ì-dire que nous avons à étudier séparément les deux cas suirants:

1. $p$ est un nombre impair. Dans ce cas le polynome $G_{a}(x)$ est précisément du degré $n$ par rapport à $x$; car le coefficient qui correspond à la puissance $x^{n}$ deriendra

$$
\frac{1}{n !}\left(s_{0}^{\prime}+\frac{1}{2}\right)
$$

et $\sigma_{0}^{\prime}$ est un nombre entier.

Cherchons dans $G_{11}(x)$ les coefficients des puissances $x^{n-2 *}$, nous aurons le développement selon les fonctions d'Fucrer

$$
\frac{1}{2} G_{n}(x)=\left(\frac{1}{2}+\sigma_{0}^{\prime}\right) E_{n}(x)+\sum_{s=1}^{\leqq} \frac{n}{2} \frac{\sigma_{2 s}^{\prime}}{(2 s) ! p^{2 s}} E_{n-2 s}(x),
$$

d'où en posant $2 n+1$ au lieu de $n$, puis supposant $x=0$ la formule récursive pour les coefficients des tangentes

$$
\begin{aligned}
& \left(\begin{array}{c}
p^{2 n+1}-1 \\
2
\end{array}+\sigma_{n}^{\prime} p^{2 n+1}\right) T_{n+1}+ \\
& \quad+\sum_{s=1}^{s=n}(-1)^{s}\left(\begin{array}{c}
2 n+1 \\
2 s
\end{array}\right) 2^{2 s} p^{2 n-2 x+1} \sigma_{2 s}^{\prime} T_{n-s+1}=(-1)^{n} 2^{2 n+1} \sigma_{2 n+1}^{\prime},
\end{aligned}
$$

où il faut supposer $n \geqq 1$.

Posons dans (17) $n=1, x=0$, nous aurons au contraire

$$
\sigma_{1}^{\prime}=\frac{p-1}{4}+\frac{\sigma_{0}^{\prime} p}{2}
$$

Introduisons maintenant dans (18), au lieu des $\sigma^{\prime}$, les sommes complómentaires $\sigma^{\prime \prime}{ }_{m}$, ce qui est permis, puis ajoutons les deux résultats ainsi ob- 
tenus, nous aurons, quelles que soient les fonctions partielles complémentaires $h_{m}(x)$ et $k_{n}(x)$,

$$
\left.\begin{array}{r}
\left(p^{2 n+1}-1\right) T_{n+1}+\sum_{s=1}^{s=n}(-1)^{s}\left(\begin{array}{c}
2 n+1 \\
2 s
\end{array}\right) 2^{2 s} p^{2 n-2 s+1} \sigma_{2 s}(p-1) T_{n-s+1}= \\
=(-1)^{n} 2^{2 n+1} \sigma_{2 n+1}(p-1) .
\end{array}\right\}
$$

Posons, dans (18),

$$
p=3, \quad \sigma^{\prime}=-1, \quad \sigma^{\prime \prime}{ }_{m}=2^{m},
$$

nous aurons les forınules particulières

$$
\begin{aligned}
& \frac{3^{2 n+1}+1}{2} T_{n+1}+\sum_{s=1}^{s=n}(-1)^{s}\left(\begin{array}{c}
2 n+1 \\
2 s
\end{array}\right) 2^{2 s} 3^{2 n-2 s+1} T_{n-s+1}=(-1)^{n-1} 2^{2 n-1} \\
& \frac{3^{2 n+2}-1}{2} T_{n+1}+\sum_{s=1}^{s=n}(-1)^{*}\left(\begin{array}{c}
2 n+1 \\
2 s
\end{array}\right) 2^{4 s} 3^{2 n-2 s+1} T_{n-s+1}=(-1)^{n} 2^{4 n+2} \text {. }
\end{aligned}
$$

2. ${ }^{\circ}$ est un nombre pair. Dans ce cas le degré de $G_{n}(x)$ ne peut pas, en vertu de (16), dépasser $n-1$; c'est-à-dire que nous aurons

$$
\sigma_{0}^{\prime}=\sigma_{0}^{\prime \prime}=-\frac{1}{2} \text {. }
$$

Or, il est facile de roir que $G_{n}(x)$ est précisément du degré $u-1$; car le coefficient qui correspond à la puissance $x^{n-1}$ deviendra

$$
\frac{1}{(n-1) ! p}\left(a^{\prime}+\frac{1}{4}\right)
$$

et $\sigma^{\prime}{ }_{1}$ est la moitié d'un nombre impair.

Nous trouvons ici le développement suivant selon les fonctions de Bernoulat

$$
\frac{1}{2} G_{n}(x)=\sum_{s=0}^{\frac{n-1}{2}} \frac{\sigma_{2 s+2}^{\prime}}{(2 s+2) ! p^{2 s+2}} B_{n-2 s-1}(x), \quad n \geqq 1,
$$

d'où en substituant $2 n+1$ au lieu de $n$, puis posant $x=0$

$$
\left.\begin{array}{c}
\frac{(n+1) p}{2^{2 n+2}} T_{n+1}+\sum_{s=0}^{s=n-1}(-1)^{n}\left(\begin{array}{l}
2 n+2 \\
2 s+2
\end{array}\right) p^{2 n-2 s} \sigma_{2 s+2}^{\prime} B_{n-s}= \\
=(-1)^{n-1}\left((n+1) p \dot{\prime}_{2 n+1}^{\prime}-\sigma_{2 n+2}^{\prime}\right)
\end{array}\right\}
$$

formule qui est valable, pourvu que $n \geqq 1$. 
Posons dans (23) $n=1, x=0$, nous aurons au contraire

$$
\sigma_{2}^{\prime}=\frac{p}{2}+2 p \sigma_{1}^{\prime}
$$

La formule (24) donnera, par le procédé ordinaire, quelles que soient les fonctions partielles complémentaires $h_{a n}(x)$ et $k_{m i}(x)$,

$$
\left.\begin{array}{rl}
\frac{(n+1) p T_{n+1}}{2^{2 n+1}}= & \sum_{s=0}^{s=n-1}(-1)^{s}\left(\begin{array}{l}
2 n+2 \\
2 s+2
\end{array}\right) p^{2 n-2 s} \sigma_{2 s+2}(p-1) B_{n-s}+ \\
& +(-1)^{n}\left((n+1) p \sigma_{2 n+1}(p-1)-\sigma_{2 n+2}(p-1)\right)
\end{array}\right)
$$

Les cas particulier $p=2$ nous conduira de nouveau à la formule (9).

\section{SUITES RÉGULik̀res de DEUXIÈme Espèce.}

Soient maintenant $f_{m}(x)$ et $g_{m}(x)$ deux fonctions partielles complémentaires quelconques qui correspondent à l'ensemble des nombres $\alpha_{s}$

et à l'ensemble adjoint

$$
1,3,5,7, \ldots, 2 p-1
$$

puis posons pour abréger

$$
\beta_{s}=1
$$

$$
f_{m}(x)=\sum_{s=1}^{s=m} \frac{t^{\prime}}{s !(2 p)^{s}} \cdot \frac{x^{m-s}}{(m-s) !}, g_{m}(x)=\sum_{s=1}^{s=m} \frac{t^{\prime \prime}}{s !(2 p)^{s}} \cdot \frac{x^{m-s}}{(m-s) !},
$$

nous aurons pour les sommes complémeritaires

d'où particulièrement

$$
t^{\prime}+t^{\prime \prime}{ }_{m}=t_{m}(p)
$$

$$
t_{0}^{\prime}=t^{\prime \prime}=\frac{p}{2}
$$

Appliquons ensuite la première des formules (7) du paragraphe IX, il résulte le théorème:

I. Soit $f_{m}(x)$ une fonction partielle quelconque formée des ensembles (1) 
et (2), les fonctions

$$
F_{n}(x)=\frac{B_{n}\left(p x-\frac{1}{2}\right)}{p^{n-1}}+f_{n-1}(x), \quad n \geqq 1
$$

sont toutes des polynomes réguliers.

Remarquons que le polynome $B_{n}\left(p x-\frac{1}{2}\right)$ ne contient que des puissances de la forme $x^{n-2 s}$, nous aurons

$$
\frac{1}{2} F_{n}(x)=\sum_{q=0}^{\leqq \frac{n}{2}} \frac{t_{2 q}^{\prime}}{(2 q) !(2 p)^{2 q}} B_{n-2 q}(x),
$$

d'où, en introduisant $2 n$ au lieu de $n$, puis posant $x=0$, la formule récursive

$$
\frac{p}{2}\left(2^{2 n}-2\right) B_{n}+\sum_{q=0}^{q=n-1}(-1)^{q}\left(\begin{array}{l}
2 n \\
2 q
\end{array}\right)(2 p)^{2 n-2 q} \ell_{2 q} B_{n-q}=(-1)^{n}\left(t_{2 n}^{\prime}-2 n p t_{2 n-1}^{\prime}\right),
$$

ce qui donnera, quelles que soient les fonctions partielles complémentaires que nous venons d'appliquer,

$$
\left.\begin{array}{rl}
p\left(2^{2 n}-2\right) B_{n}+\sum_{q=0}^{q=n-1}(-1)^{q}\left(\begin{array}{l}
2 \\
2 \\
2
\end{array}\right) & (2 p)^{2 n-2 q} t_{2 q}(p) B_{n-q}= \\
& =(-1)^{n}\left(t_{2 n}(p)-2 n p t_{2 n-1}(p)\right)
\end{array}\right\}
$$

Soit particulièrement $p=1$, nous retrouvons la formule (9) du paragraphe $\mathrm{X}$, tandis que les hypothèses

$$
p=2, \quad t_{m}^{\prime}=1, \quad t_{m}^{\prime \prime}=3^{m}
$$

donnent, en vertu de (8),

$$
\begin{gathered}
\frac{\left(2^{2 n}+2\right) n T_{n}}{2^{2 n-1}}=\sum_{q=1}^{q=n-1}(-1)^{q-1}\left(\begin{array}{l}
2 n \\
2 q
\end{array}\right) 2^{4 n-4 q} B_{n-q}-(-1)^{n}(4 n-1) \\
\frac{\left(2^{2 n}+2\right) n T_{n}}{2^{2 n-1}}=\sum_{q=1}^{q=n-1}(-1)^{q-1}\left(\begin{array}{l}
2 n \\
2 q
\end{array}\right) 3^{2 q} 2^{4 n-4 q} B_{n-q}-(-1)^{n}(4 n-3) 3^{2 n-1} .
\end{gathered}
$$

Étudions maintenant le cas, où l'ensemble adjoint de (1) se détermine par les nombres

$$
\beta_{s}=(-1)^{s}, \quad \alpha_{s}=2 s+1,
$$


puis désignons par $h_{m}(x)$ et $k_{m}(x)$ deux fonctions partielles complémentaires formées de ces ensembles, nous aurons en posant

$$
h_{m}(x)=\sum_{s=0}^{s=m} \frac{\tau_{s}^{\prime}}{s !(2 p)^{s}} \cdot \frac{x^{m-s}}{(m-s) !}, \quad k_{m}(x)=\sum_{s=0}^{s=m} \frac{\tau^{\prime \prime} s}{s !(2 p)^{s}} \cdot \frac{x^{m-s}}{(m-s) !}
$$

pour les sommes complémentaires $\tau^{\prime}{ }_{m}$ et $\tau^{\prime \prime}{ }_{m}$ la condition suivante

$$
\tau^{\prime}{ }_{m}+\tau^{\prime \prime}{ }_{m}=(-1)^{p-1} \tau_{n}(p)
$$

Cela posé, la dernière des formules (7) du paragraphe IX donnera le théorème:

[I. Soit $h_{m}(x)$ une fonction partielle quelconque, formée des ensembles (1) et (12), les fonctions

$$
G_{n}(x)=\frac{E_{n}\left(p x-\frac{1}{2}\right)}{p^{n}}-h_{n}(x)
$$

sont toutes des polynomes réguliers.

L'équation fonctionnelle correspondante, savoir

$$
(-1)^{n+p} G_{n}(-x-1)=G_{n}(x)
$$

dépendant de la parité de $p$, nous avons à étudier séparément les deux cas suivants:

$10^{\circ} p$ est un nombre pair. Les mêmes réflexions que dans le paragraphe précédent montrent que $G_{n}(x)$ est toujours un polynome du degré $n$; remarquons ensuite que $E_{n}\left(p x-\frac{1}{2}\right)$ ne contient que des puissances de $x$ de la forme $x^{n-2 x}$, nous aurons le développement

$$
\frac{1}{2} G_{n}(x)=-\sum_{s=0}^{\leqq \frac{n}{2}} \frac{\dot{r}_{2 s+1}^{\prime}}{(2 s+1) !(2 p)^{2 s+1}} B_{n-2, s}(x) .
$$

Introduisons ensuite dans (17) $2 u$ au lieu de $n$, puis posons $x=0$, il résulte la formule récursive

$$
\left.\begin{array}{r}
\left(n+\frac{1}{2}\right) p E_{n}=\sum_{s=0}^{s=n-1}(-1)^{x}\left(\begin{array}{l}
2 n+1 \\
2 s+1
\end{array}\right)(2 p)^{2 n-2 x} \tau_{2 s+1}^{\prime} B_{n-s}- \\
-(-1)^{n}\left((2 n+1) p \tau_{2 n}^{\prime}-\div_{n n+1}^{\prime}\right),
\end{array}\right\}
$$


d'où, en appliquant la méthode ordinaire,

$$
\left.\begin{array}{r}
(2 n+1) p E_{n}+\sum_{s=0}^{s=n-1}(-1)^{s}\left(\begin{array}{l}
2 n+1 \\
2 s+1
\end{array}\right)(2 p)^{2 n-2 s} s_{2 s+1}(p) B_{n-s}= \\
=(-1)^{n-1}\left(\tau_{3_{n+1}}(p)-(2 n+1) p \tau_{2 n}(p)\right),
\end{array}\right\}
$$

et nous trouvons toujours cette formule, quelles que soient les deux fonctions partielles complémentaires $h_{m}(x)$ et $k_{m}(x)$.

Posons, dans (18),

$$
p=2, \quad \tau_{m}^{\prime}=1, \quad \tau^{\prime \prime}=-3^{m},
$$

nous aurons respectivement

$$
\begin{aligned}
& (2 n+1) E_{n}=\sum_{s=0}^{s=n-1}(-1)^{s}\left(\begin{array}{l}
2 n+1 \\
2 s+1
\end{array}\right) 2^{n-4 s} B_{n-s}+(-1)^{n}(4 n+1) \\
& (2 n+1) E_{n}+\sum_{s=0}^{s=n-1}(-1)^{s}\left(\begin{array}{l}
2 n+1 \\
2 s+1
\end{array}\right) 3^{2 n+1} 2^{4 n-4 s} B_{n-s}=(-1)^{n-1}(4 n-1) 3^{2 n} .
\end{aligned}
$$

2. $p$ est un nombre impair. Dans ce cas $G_{n}(x)$ est un polynome du degré $n-1$, de sorte que nous aurons ici

$$
\frac{1}{2} G_{n}(x)=-\sum_{s=0}^{\leqq \frac{n-1}{2}} \frac{\tau_{2 s+1}^{\prime}}{(2 s+1) !(2 p)^{2 s+1}} E_{n-2 s-1}(x), \quad n \geqq 1,
$$

ce qui donnera en substituant $2 n$ au lieu de $n$ et en posant $x=0$

$$
\begin{gathered}
\frac{1}{2} E_{n}=\sum_{s=0}^{s=n-1}(-1)^{s}\left(\begin{array}{c}
2 n \\
2 s+1
\end{array}\right) p^{2 n-2 s-1} \tau_{2 s+1}^{\prime} T_{n-s}+(-1)^{n} \tau_{2 n}^{\prime} \\
E_{n}=\sum_{s=0}^{s=n-1}(-1)^{s}\left(\begin{array}{c}
2 n \\
2 s+1
\end{array}\right) p^{2 n-9 s-1}-_{2 s+1}(p) T_{n-s}+(-1)^{n} \tau_{2 n}(p) .
\end{gathered}
$$

Posons $p=1$, nous aurons la formule d'EuLen $\left(^{*}\right)$

$$
E_{n}=\sum_{s=0}^{s=n-1}(-1)^{x}\left(\begin{array}{c}
2 n \\
2 s+1
\end{array}\right) T_{n-s}+(-1)^{n},
$$

ce qui est précisément la formule (12) du paragraphe 1.

(*) Opuscula analytica, t. II, pp. 269-270. Saint-Pétersbourg, 1785. 
XII. SuItes RÉGULIÈres DE TROISIÈme ESPÈce.

Il est très curieux, ce me semble, que les formules (8) et (9) du paragraphe IX nous permettent de généraliser les résultats obtenus dans les deux paragraphes précédents.

En effet, désignons par $m>1$ un entier quelconque du reste, par

$$
\alpha_{1} \alpha_{2} \alpha_{3} \ldots \alpha_{\mu}, \quad \mu=\varphi(m)
$$

les positifs entiers plus petits que $m$ et premiers avec $m$ et déterminons l'ensemble adjoint de sorte que

$$
\beta_{s}=1
$$

nous aurons pour les coefticients des deux fonctions partielles complémentaires quelconques

$$
f_{n}(x)=\sum_{q=0}^{q=n} \frac{s_{q}^{\prime}}{q ! m^{q}} \cdot \frac{x^{n-q}}{(n-q) !}, \quad g_{n}(x)=\sum_{q=0}^{q=n} \frac{s^{\prime \prime}{ }_{q}}{q ! m^{q}} \cdot \frac{x^{n-q}}{(n-q) !}
$$

les conditions suivantes

$$
s_{q}^{\prime}+s_{q}^{\prime \prime}=s_{q}=\sum_{s=1}^{s=\mu} \alpha_{q}^{q} .
$$

Cela posé, nous aurons le théorème suivant:

I. Soit $m>1$ un entier quelconque du reste, et soit $f_{n}(x)$ une fonction partielle quelconque, formée des ensembles (1) et (2), les fonctions

$$
\Phi_{n}(x)=F_{n}(x)+f_{n-1}(x), \quad n \geqq 1,
$$

où $F_{n}(x)$ est la fonction définie par la formule (10) du paragraphe IX, sont toutes des polynomes réguliers.

Nous aurous immédiatement le développement

$$
\frac{1}{2} \Phi_{n}(x)=\sum_{q=0}^{\leqq \frac{n}{2}} \frac{s_{2 q}^{\prime}}{(2 q) ! m^{2 q}} B_{n-2 q}(x),
$$

d'où en remplaçant $n$ par $2 n$, puis posant $x=0$, la formule récursive très 
curieuse

$$
\left.\begin{array}{l}
\frac{(-1)^{r} m \varphi_{2 n-1}(m)}{2} B_{n}+(-1)^{n}\left(s_{2 n}^{\prime}-m n s_{2 n-1}^{\prime}\right)= \\
=\sum_{q=0}^{q=n-1}(-1)^{q}\left(\begin{array}{l}
2 \\
2 \\
2 q
\end{array}\right) s_{2_{q}}^{\prime} m^{2 n-2 q} B_{n-q} .
\end{array}\right\}
$$

Introduisons dans (7) au lieu de $s_{q}^{\prime}$ les sommes correspondantes, puis additionnons les deux formules ainsi obtenues, nous aurons toujours, quelles que soient les fonctions partielles complémentaires appliquées:

$$
\left.\begin{array}{rl}
(-1)^{n} m \varphi_{2 n-1}(m) B_{n}+(-1)^{n} & \left(s_{2 n}-m n s_{2 n-1}\right)= \\
= & \sum_{q=0}^{q=n-1}(-1)^{q}\left(\begin{array}{l}
2 \\
2 \\
2 q
\end{array}\right) m^{2 n-2 q} s_{2 q} B_{n-q} .
\end{array}\right\}
$$

Posons encore dans (7)

$$
m=6, \quad r=2, \quad s_{n}^{\prime}=1, \quad s_{n}^{\prime \prime}=5^{n}
$$

il résultent les formules récursives

$$
\begin{aligned}
& \left(3^{2 n}-3\right)\left(2^{2 n-1}-1\right) B_{n}=\sum_{q=0}^{q=n-1}(-1)^{q}\left(\begin{array}{l}
2 \\
2 \\
2 q
\end{array}\right) 6^{2 n-2 q} B_{n-q}+(-1)^{n}(6 n-1) \\
& \left.\begin{array}{rl}
\left(3^{2 n}-3\right)\left(2^{2 n-1}-1\right) B_{n}= & \sum_{q=0}^{q=n-1}(-1)^{q}\left(\begin{array}{l}
2 \\
2 \\
2 q
\end{array}\right) 5^{2 q} 6^{2 n-2 q} B_{n-q}+ \\
& +(-1)^{n}(6 n-5) 5^{2 n-1} .
\end{array}\right\}
\end{aligned}
$$

Quant à l'application des formules (9) et (11) du paragraphe IX, nous supposons $m$ impair et nous remplaçons l'ensemble (2) par cet autre

$$
\beta_{s}=(-1)^{\alpha_{s}}
$$

Soient ensuite $h_{n}(x)$ et $k_{n}(x)$ deux fonctions partielles complémentaires formées de ces deux ensembles (1) et (11), nous posons

$$
h_{n}(x)=\sum_{q=0}^{q=\bar{\Sigma}^{n}} \frac{\sigma_{q}^{\prime}}{q ! m^{q}} \cdot \frac{x^{n-q}}{(n-q) !}, \quad k_{n}(x)=\sum_{q=0}^{q=n} \frac{\sigma^{\prime \prime} q}{q ! m^{q}} \cdot \frac{x^{n-q}}{(n-q) !},
$$

ce qui donnera

$$
\sigma^{\prime}+\sigma_{q}^{\prime \prime}=\sigma_{q}=\sum_{s=1}^{s=\mu}(-1)^{\alpha_{*}} \alpha_{z}^{q}
$$


d'où particulièrement pour $q=0$

$$
\sigma_{q}^{\prime}+r_{q}^{\prime \prime}=0
$$

Cela posé, nous aurons le théorème suivant:

II. Soit $m>1$ un nombre entier impair quelconque du reste, et soit $h_{n}(x)$ une fonction partielle quelconque formée des ensembles (1) et (11), les fonctions

$$
\Psi_{n}(x)=G_{n}(x)+h_{n}(x)
$$

où $G_{n}(x)$ est la fonction définie par la formule (11) du paragraphe IX, sont toutes des polynomes réguliers.

Dans ce cas nous aurons le développement

$$
\frac{1}{2} \Psi_{n}(x)=\sum_{q=0}^{\frac{n}{2}} \frac{\sigma_{2 q}^{\prime}}{(2 q) ! m^{2 q}} E_{n-2 q}(x)
$$

d'où, en remplaçant $n$ par $2 n+1$, puis posant $x=0$, la formule récursive curieuse

$$
\left.\begin{array}{r}
\frac{(-1)^{r}}{2} \varphi_{2 n+1}(m) T_{n+1}=\sum_{q=0}^{q=n}(-1)^{x}\left(\begin{array}{c}
2 n+1 \\
2 q
\end{array}\right) 2^{2 q} m^{2 n-2 q+1} \sigma_{{ }^{\prime} q} T_{n-q+1}- \\
-(-1)^{n} 2^{2 n+1} \sigma^{\prime}{ }_{2 n+1},
\end{array}\right\}
$$

de sorte que nous aurons, en vertu de (14),

$$
\left.\begin{array}{r}
(-1)^{\prime} \varphi_{2 n+1}(m) T_{n+1}^{T}=\sum_{q=0}^{q=n}(-1)^{q}\left(\begin{array}{c}
2 n+1 \\
2 q
\end{array}\right) 2^{2 n} m^{2 n-2 q+1} \sigma_{2 q} T_{n-q+1}- \\
-(-1)^{n} 2^{2 n+1} \sigma_{2 n+1} .
\end{array}\right\}
$$

Soit $m$ un nombre premier, les formules récursives générales que nous venons de développer coïncilent avec celles obtenues dans le paragraphe $\mathrm{X}$. 


\section{QUATRIÈME PARTIE.}

\section{Applications diverses.}

XIII. SUR DEUX ÉQUations aLGÉbriques.

KroneCKen $\left({ }^{*}\right)$, dans ses recherches remarquables, trouve pour le nombre général $B_{n}$ de Bernoull deux expressions à l'aide des sommes des puissances des racines de deux équations algébriques.

Or, il est très facile de trouver d'autres expressions de ce genre et plus simples que celles de KroneGKer.

A cet effet, prenons pour point de départ la formule d'Eulen, savoir la formule (9) du paragraphe $\mathrm{V}$

$$
\sum_{r=0}^{r=n-1} \frac{(-1)^{r}}{(2 r+1) !} \cdot \frac{2^{2 n-2 r-1} B_{n-r}}{(2 n-2 r) !}+\frac{(-1)^{n} n}{(2 n+1) !}=0
$$

puis désignons par $s_{q}$ la somme des $q$-ièmes puissances de $m$ racines de l'équation algébrique

$$
\frac{x^{m}}{1 !}-\frac{x^{m-1}}{3 !}+\frac{x^{m-2}}{5 !}-\cdots=0
$$

la formule de Newton domera, pour $1 \leqq n \leqq m$

$$
\sum_{r=0}^{n-1} \frac{(-1)^{r} s_{n-r}}{(2 r+1) !}+\frac{(-1)^{n} n}{(2 n+1) !}=0,
$$

d'où en vertu de (1)

$$
s_{n}=\frac{2^{2 n-1} B_{n}}{(2 n) !}, \quad 1 \leqq n \leqq m .
$$

Appliquons ensuite la formule récursive de $J_{A C O B I}\left({ }^{*}\right)$

$$
\sum_{r=0}^{r=n-1}(-1)^{r}(2 r+2) ! \cdot \frac{B_{n-r}}{(2 n-2 r) !}+\frac{(-1)^{n} n}{(2 n+2) !}=0,
$$

(*) Journal de Mathèmatiques pures et appliquées (2), t. 1, pp. 385-391; 1856.

${ }^{(* *)}$ Journal de Crelle, t. 12, p. 265; 1834.

Annali di Matematica, Serie III, Tomo XXII. 
ce qui n'est autre chose que l'expression de $B_{2 n+2}(-1)$ tirée directement de la formule (2) du paragraphe I, puis désignons par $s_{q}^{\prime}$ la somme des $q$-ièmes puissances des $m$ racines de l'équation algébrique

$$
\frac{x^{2 n}}{2 !}-\frac{x^{m-1}}{4 !}+\frac{x^{m-2}}{6 !}-\cdots=0
$$

nous aurons de même, en vertu de la formule de Nwwton,

$$
\sum_{r=0}^{r=n-1}(-1)^{r} s_{n-r}+\frac{(-1)^{n} n}{(2 r+2) !}=0, \quad 1 \leqq n \leqq m
$$

d'où, en vertu de (5),

$$
s_{n}^{\prime}=\frac{B_{n}}{(2 n) !}, \quad 1 \leqq n \leqq n
$$

Cela posé, les formules (4) et (8) doment, pour les deux équations algébriques (4) et (8), la relation

$$
s_{n}=Q^{m-1} s_{n}^{\prime}, \quad 1 \leqq n \leqq m
$$

j'ignore comment démontrer directement cette formule curieuse.

M. Mande $\left(^{*}\right)$ a trouvé, pour les nombres (4), la formule récursive (3) sans remarquer qu'elle appartient à Eocks.

\section{Sur un théorème de Syluester.}

Il nous reste encore à montrer comment on pourrait tirer directement de nos formules précédentes quelques propriétés essentielles des nombres de Burxougli.

En prenier lieu la formule de Hosver, savoir la formule (10) du paragraphe $V$, domera, par la conclusion de $n$ a $n+1$, une expression de la forme

$$
2.3 .5 \cdot 7 \ldots(2 n+1) B_{n}=2 k+1,
$$

où $k$ est un entier non négatif; c'est-à-dire rue nous pourons toujours ad-

(*) Sitaungsherichte der Wiener Akarlemie, t. 94, I, p. 953; 1886. 
mettre

$$
B_{n}=\frac{a_{n}}{2 b_{n}}
$$

où $\dot{a}_{n}$ et $b_{n}$ sont des entiers impairs premiers entre eux.

Désignons ensuite par $2 G_{n}$ le plus petit dénominateur commun des $n$ premiers nombres de Bernoulci, $G_{n}$ est toujours un nombre impair.

Appliquons ensuite la formule (9) du paragraphe $X$, savoir

$$
\frac{n T_{n}}{\overline{2}^{22 n-2}}=\sum_{q=1}^{q=n-1}(-1)^{q-1}\left(\begin{array}{l}
2 n \\
2 q
\end{array}\right) 2^{2 n-2 q} B_{n-q}+(-1)^{n-1}(2 n-1),
$$

le second membre est, en vertu de (1), un entier; multiplions par $G_{n-1}$, nous verrons que ce nombre est impair, de sorte que nous aurons

$$
n T_{n}=2^{2 n-2}(2 K+1)
$$

où $K$ est un entier non négatif.

Cela posé, nous aurons immédiatement le théorème suivant indiqué par WoRPITrKKY (*):

I. Soit l'indice $n$ un nombre de la forme $2^{p}(2 q+1)$, nous aurons pour le n-ième coefficient des tangentes une expression de la forme

$$
T_{n}=2^{2 n-p-2}(2 r+1) .
$$

En dernier lieu, nous avons à appliquer la formule (8) du paragraphe $X$, savoir

$$
\left.\begin{array}{rl}
p\left(p^{2 n}-1\right) B_{n}+\sum_{q=1}^{q=n-1}(-1)^{q}\left(\begin{array}{l}
2 \\
2 \\
2 q
\end{array}\right) p^{2 n-2 q} s_{2 q}(p-1) B_{n-q}= \\
=(-1)^{n}\left(s_{2 n}(p-1)-n p s_{2 n-1}(p-1)\right)
\end{array}\right\}
$$

combinée avec un résultat obtenu pour la somme

$$
s_{n}(q)=1^{n}+2^{n}+3^{n}+\cdots+q^{n} .
$$

En effet, posons dans l'expression de $B_{n+1}(x)$ successivement $x=q$, $x=0$, puis soustrayons, il en résulte la formule classique

$$
s_{n}(q)=\frac{q^{n+1}}{n+1}+\frac{q^{n}}{2}+\sum_{s=1}^{\leqq \frac{n}{2}} \frac{(-1)^{s-1}}{n+1}\left(\begin{array}{c}
n+1 \\
2 s
\end{array}\right) B_{s} q^{n-2 s+1}
$$

(*) Journal de Crelle, t. 94, p. 232; 1883. 
posons ensuite dans cette formule $q=1,2,3, \ldots, p-1$, puis ajoutons tous les résultats ainsi obtenus, nous aurons

$$
\sum_{q=1}^{q=p-1} s_{n}(q)=\frac{s_{n+1}(p-1)}{n+1}+\frac{s_{y}(p-1)}{2}+\sum_{s=1}^{\leqq} \frac{n}{2} \frac{(-1)^{s-1}}{n+1}\left(\begin{array}{c}
n+1 \\
2 s
\end{array}\right) B_{s} s_{n-2 s+1}(p-1) .
$$

Or, nous aurons pour le premier membre de cette formule l'expression

$$
(p-1) 1^{n}+(p-2) 2^{n}+\cdots+1 .(p-1)^{n}=p s_{n}(p-1)-s_{n+1}(p-1) ;
$$

remplaçons enfin $n$ par $2 n-1$, il en résulte

$$
\begin{aligned}
& \sum_{q=1}^{q=n-1}(-1)^{q}\left(\begin{array}{l}
2 \\
2 \\
2
\end{array}\right) s_{2_{q}}(p-1) B_{n-q}= \\
& =(-1)^{n}\left((2 n+1) s_{2 n}(p-1)-(2 n p-n) s_{2 n-1}(p-1)\right)
\end{aligned}
$$

d'où, en soustrayant les formules (4) et (6), puis multipliant par $p^{n}$ et divisant par $2 n$, nous trouvons la formule

$$
\left.\begin{array}{c}
\frac{p^{n+1}\left(p^{2 n}-1\right)}{2 n} B_{n}+\sum_{q=1}^{q=n-1}(-1)^{q}\left(\begin{array}{c}
2 n-1 \\
2 q
\end{array}\right) s_{2_{q}}(p-1) \frac{p^{n}\left(p^{2 n-2 q}-1\right)}{2 n-2 q} B_{n-q}= \\
=(-1)^{n}\left(\frac{p^{n}(p-1)}{2} s_{2 n-1}(p-1)-p^{n} s_{2 n}(p-1)\right)
\end{array}\right\}
$$

Remarquons maintenant que l'expression

$$
\frac{p^{2}\left(p^{2}-1\right)}{2} B_{1}=\frac{p^{2}(p+1)(p-1)}{12}
$$

est toujours un nombre entier, pourvu que $p$ le soit, la formule (7) donnera par la conclusion de $n$ à $n+1$, le théorème suivant:

II. Soit $p$ un nombre entier quelconque, l'expression

$$
C_{n}=\frac{p^{n+1}\left(p^{2 n}-1\right)}{2 n} B_{n}
$$

est toujours un nombre entier.

On sait que SyLvester (*) a démontré que l'expression

$$
\frac{p^{2 n}\left(p^{2 n}-1\right)}{2 n} B_{n}
$$

est un nombre entier, résultat qui est retrouvé par Lrpschiтz (**).

(*) Philosophical Magazine, février 1861.

(**) Journal de Crelle, t. 96, p. $2 ; 1884$. 
Soit $p$ un nombre de la forme $6 q \pm 1$, nous verrons, en vertu de (8), que l'expression

$$
\frac{p^{n-1}\left(p^{2 n}-1\right)}{2 n} B_{n}
$$

est toujours un nombre entier.

Posons particulièrement, dans (9), $p=2$, nous verrons, en vertu de (1), que le nombre

$$
A_{n}=2\left(2^{2 n}-1\right) B_{n}
$$

est toujours un nombre entier impair, résultat qui était connu déjà par Euler $\left(^{*}\right)$.

Remarquons l'identité

$$
n T_{n}=2^{2 n-2} A_{n},
$$

le théorème de WonprTzky est évident et se présente comme une conséquence immédiate des formules eulériennes susdites.

(*) Institutiones calculi differentialis, pp. 495-497. Saint-Pétersbourg, 1755. 